Draft Version April 15, 2021

Preprint typeset using $\mathrm{IAT}_{\mathrm{E}} \mathrm{X}$ style emulateapj v. 03/07/07

\title{
CHEMICAL ABUNDANCES OF PLANETARY NEBULAE IN THE SUBSTRUCTURES OF M31*
}

\author{
Xuan Fang ${ }^{1}$, Rubén García-Benito ${ }^{1}$, Martín A. Guerrero ${ }^{1}$, Xiaowei Liu ${ }^{2,3}$, Haibo Yuan $^{3,4}$, \\ YONG ZHANG ${ }^{5}$, AND BING ZHANG ${ }^{2}$ \\ ${ }^{1}$ Instituto de Astrofísica de Andalucía (IAA-CSIC), Glorieta de la Astronomía s/n, E-18008 Granada, Spain \\ ${ }^{2}$ Department of Astronomy, School of Physics, Peking University, Beijing 100871, China \\ ${ }^{3}$ Kavli Institute for Astronomy and Astrophysics, Peking University, Beijing 100871, China \\ ${ }^{4}$ LAMOST Fellow \\ ${ }^{5}$ Department of Physics, University of Hong Kong, Pokfulam Road, Hong Kong, China \\ Draft version April 15, 2021
}

\begin{abstract}
We present deep spectroscopy of planetary nebulae (PNe) that are associated with the substructures of the Andromeda Galaxy (M31). The spectra were obtained with the OSIRIS spectrograph on the $10.4 \mathrm{~m}$ GTC. Seven targets were selected for the observations, three in the Northern Spur and four associated with the Giant Stream. The most distant target in our sample, with a rectified galactocentric distance $\geq 100 \mathrm{kpc}$, was the first PN discovered in the outer streams of M31. The [O III] $\lambda 4363$ auroral line is well detected in the spectra of all targets, enabling electron temperature determination. Ionic abundances are derived based on the $[\mathrm{O}$ III] temperatures, and elemental abundances of helium, nitrogen, oxygen, neon, sulfur, and argon are estimated. The relatively low $\mathrm{N} / \mathrm{O}$ and $\mathrm{He} / \mathrm{H}$ ratios as well as abundance ratios of $\alpha$-elements indicate that our target PNe might belong to populations as old as $\sim 2$ Gyr. Our PN sample, including the current seven and the previous three observed by Fang et al., have rather homogeneous oxygen abundances. The study of abundances and the spatial and kinematical properties of our sample leads to the tempting conclusion that their progenitors might belong to the same stellar population, which hints at a possibility that the Northern Spur and the Giant Stream have the same origin. This may be explained by the stellar orbit proposed by Merrett et al. Judging from the position and kinematics, we emphasize that M32 might be responsible for the two substructures. Deep spectroscopy of PNe in M32 will help to assess this hypothesis.
\end{abstract}

Subject headings: galaxies: abundances - galaxies: formation - galaxies: individual (M31) - ISM: abundances - planetary nebulae: general - stars: evolution

\section{INTRODUCTION}

Merging of smaller galaxies makes major contributions to the growth of a large galaxy (e.g., White 1978: White \& Rees 1978). The relics of such interaction can be preserved in the outskirts of galaxies and detected as various substructures such as tidal tails or stellar streams (e.g., Ibata et al. 2001a; Majewski et al. 2003). The Andromeda Galaxy (M31), being the nearest large spiral system, is probably the best candidate for studying the debris of galaxy interaction/formation. Numerous stellar substructures have been detected in its outskirts (e.g., Ibata et al. 2001b, 2007; Ferguson et al. 2002; Irwin et al. 2005: McConnachie et al. 2003, 2004, 2009). The morphologies and stellar populations of the substructures provide important clues to the assemblage history of the galaxy. However, due to the intrinsic faintness and the vast spatial extension of these substructures, a comprehensive survey of the stars therein is difficult. As descendants of the low- and intermediate-mass stars, planetary nebulae $(\mathrm{PNe})$ are ubiquitous in the universe and excellent tracers to study the chemistry, kinematics and stellar contents of the substructures. Being bright in narrow emission lines, $\mathrm{PNe}$ are easily detectable at the distance of M31 (785 kpc; McConnachie et al. 2005), and their

\footnotetext{
Electronic address: fangx@iaa.es

* Based on observations made with the Gran Telescopio Canarias, installed at the Spanish Observatorio del Roque de los Muchachos of the Instituto de Astrofísica de Canarias, in the island of La Palma. These observations are associated with program No. GTC55-14B.
}

spectra can be used to measure radial velocities and derive accurate abundances of elements such as $\mathrm{He}, \mathrm{O}, \mathrm{C}$, $\mathrm{N}, \mathrm{Ne}, \mathrm{Ar}$ and $\mathrm{S}$.

Among all substructures found in M31, the southern stellar stream (also known as the Giant Stream; Ibata et al. 2001b Caldwell et al. 2010) and the Northern Spur are the most prominent. The Northern Spur was originally observed to lie in the direction of M31's gaseous warp (Newton \& Emerson 1977), and Ferguson et al. (2002) found enhancement in both stellar density and metallicity in the Northern Spur through a panoramic survey of the red giant branch (RGB) stars in M31. The Giant Stream was discovered by Ibata et al. (2001b) as a stream of metal-rich stars within the halo of M31. Ferguson et al. (2002) proposed the possible connection between the Giant Stream and the Northern Spur. Merrett et al. (2003) presented a three-dimensional orbit for the stellar streams that connects the two substructures by studying the kinematics of $\sim 20 \mathrm{PNe}$ in the disk of M31. It has been suggested that dwarf galaxies such as M32 and NGC 205 might be responsible for the two substructures (e.g., Ibata et al. 2001b; Merrett et al. 2003) mainly because the two satellites are spatially associated with the Stream.

Today the exact origin of the Giant Stream and the Northern Spur of M31 is still unclear, and observational data for them are scarce. Spectroscopy of the PNe in these two substructures can provide accurate chemical information to shed light on this problem. With this purpose, Fang et al. (2013, hereafter Paper I) carried out 
spectroscopic observations of three PNe in the Northern Spur using the Double Spectrograph on the $5.1 \mathrm{~m}$ Hale telescope at Palomar Observatory. The authors found relatively high oxygen abundance in one of the three targets compared to the disk sample of Kwitter et al. (2012) at similar galactocentric distance, a result that might be in line with the paradigm of tidal disruption of M31 satellites. Balick et al. (2013) observed two halo/outer-disk PNe in M31 using the $10.4 \mathrm{~m}$ Gran Telescopio Canarias (GTC). The authors invoked starburst triggered by M31M33 encounter to explain the unexpected high oxygen abundances found in these two PNe. More recent observations of outskirt PNe by Corradi et al. (2015) support the current view that the external regions of M31 are the result of complex interactions and merger processes.

We have carried out deep spectroscopy of seven PNe in the Northern Spur and the Giant Stream of M31 using the $10.4 \mathrm{~m}$ GTC. The purpose of these observations is to derive reliable elemental abundances of $\mathrm{He}$, $\mathrm{O}, \mathrm{N}, \mathrm{Ne}, \mathrm{Ar}$ and $\mathrm{S}$. We expect excellent detection of the temperature-sensitive auroral lines of heavy elements (i.e., [O III] $\lambda 4363$, and the even fainter [N II] $\lambda 5755$ line), given the high light-collection efficiency of GTC and excellent observing conditions at Observatorio de Roque de los Muchachos (ORM). This paper is a follow-up project of Paper I to investigate the origin of M31 substructures by studying the chemistry of PNe therein. Section 2 presents the observations and data reduction. Line fluxes, plasma diagnostics and abundance determinations are given in Section 3. We discuss the spatial distribution and kinematics, abundance correlations, stellar population of the PN progenitors, the radial distribution of oxygen, and the origin of the substructures in Section 4 . Finally, we make a summary and present conclusions in Section 5.

\section{OBSERVATIONS AND DATA REDUCTION}

\subsection{Target Selection}

We selected seven PNe located in or associated with the Northern Spur and the extension of the Giant Stream. Of the seven PNe in our sample, six were selected from the catalog of Merrett et al. (2006), and the other one was identified by the Large Sky Area Multi-Object Fiber Spectroscopic Telescope (LAMOST; Su et al. 1998) during its early commissioning phase (Yuan et al. 2010). Three PNe of our sample have been identified as being in the Northern Spur by Merrett et al. (2006), and the other four are kinematically associated with the Giant Stream. Their projected galactocentric distances range from $0.7-$ 50.2 kpc. The coordinates (right ascension - R.A., and declination - Dec.), apparent magnitudes $m(\lambda 5007)$, radial velocities, and sky-projected galactocentric distances (in kpc) are presented in Table 11. The six PNe selected from Merrett et al. (2006) are relatively bright, with $m(\lambda 5007) \sim 20.4-21.3$. If the bright mercury line (Hg I $\lambda 4358)$ exists, the heliocentric velocity of M31 ( $-300 \mathrm{~km} \mathrm{~s}^{-1}$ ) could make the [O III] $\lambda 4363$ auroral line in a PN spectrum difficult to resolve (e.g., Kwitter et al. 2012 Paper I), and thus a loss of important temperature diagnostic for this target. Given the excellent observing condition at ORM in La Palma, where the mercury line is faint, target selection were not confined by radial velocities but mainly based on the brightness. The PN

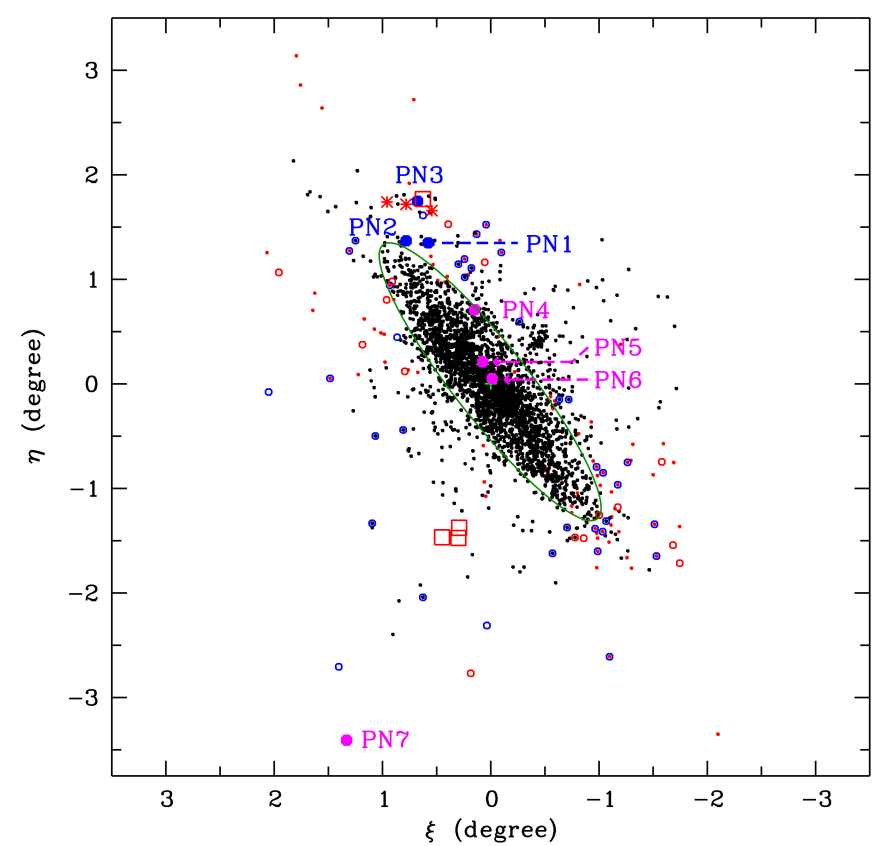

Fig. 1. - M31 PNe discovered by Merrett et al. (2006 black dots; including those searched by Hurley-Keller et al. 2004), Kniazev et al. (2014, red dots) and LAMOS'T (blue open circles, Yuan et al. 2010 red open circles, unpublished). The three Northern Spur PNe observed in Paper I are indicated by red asterisks. The seven PNe in this study, three in the Northern Spur (blue filled circles) and four associated to the extension of the Giant Steam (magenta filled circles), are named PN1 to 7. Red open squares are the Hubble Space Telescope Advanced Camera for Surveys fields studied by Bernard et al. (2015), one at the Northern Spur and three at the Giant Stream. The coordinates $\xi$ and $\eta$ represent the M31-based reference frame defined by Huchra et al. (1991). The green ellipse is a disk with a $2^{\circ}$ radius $(27.4 \mathrm{kpc})$ around the center of M31, assuming an inclination angle of $77^{\circ} .7$ and a position angle of $37^{\circ} .7$ (de Vaucouleurs 1958) for the M31 disk.

newly discovered by LAMOST, which is located $\sim 3.65$ from the center of M31 (Figure 1), is of particular interest because it is both spatially and kinematically related to the Giant Stream, and is also the first PN discovered in the outer extension of this stream. These targets are hereafter referred to PN1-7 (Table 1).

\subsection{Observations}

Spectroscopy of the seven $\mathrm{PNe}$ was carried out with OSIRIS (Optical System for Imaging and lowintermediate-Resolution Integrated Spectroscopy) on the $10.4 \mathrm{~m}$ GTC at ORM in La Palma, Spain. The observations were obtained on seven nights, from of August 23 to August 29, 2014 in service mode. The excellent observing conditions at ORM allow photometric and clear nights with seeing $0 . .5-1^{\prime \prime} 0$. The $1^{\prime \prime}$ wide long slit and the grism $\mathrm{R} 1000 \mathrm{~B}$ were used. The R1000B is a 1000 lines $\mathrm{mm}^{-1}$ grism centered at $5455 \AA$. The detector of OSIRIS consists of a mosaic of two Marconi CCDs (named CCD1 and CCD2) with $2048 \times 4096$ pixels each. The pixel physical size is $15 \mu \mathrm{m}$, which corresponds to a scale of 0 "! 127 on the sky. The OSIRIS standard observing mode uses 0 ". 254 binned pixels. This instrument setup enabled us to perform spectroscopy over the wavelength range $\sim 3630$ $7760 \AA$ with a spectral resolution of $\sim 6 \AA$ (full width at half-maximum, FWHM) at $2.06 \AA$ pixel $^{-1}$.

Except PN6, which is close to the center of M31 
TABLE 1

Properties and Observing Log of the Seven PNe

\begin{tabular}{lccrrrc}
\hline \hline PN ID $^{a}$ & $\begin{array}{c}\text { R.A. } \\
(\mathrm{J} 2000.0)\end{array}$ & $\begin{array}{c}\text { Decl. } \\
(\mathrm{J} 2000.0)\end{array}$ & $m(\lambda 5007)^{b}$ & $\begin{array}{r}v_{\text {helio }} \\
\left(\mathrm{km} \mathrm{s}^{-1}\right)\end{array}$ & $\begin{array}{r}R_{\text {gal }}{ }^{c} \\
(\mathrm{kpc})\end{array}$ & $\begin{array}{c}\text { GTC Exp. } \\
(\mathrm{s})\end{array}$ \\
\hline PN1 (M2445) & $0: 45: 52.8$ & $42: 36: 52.9$ & 20.65 & -149.1 & 20.1 & $4 \times 1250$ \\
PN2 (M2451) & $0: 46: 59.3$ & $42: 37: 58.2$ & 20.81 & -80.8 & 21.6 & $4 \times 1250$ \\
PN3 (M2427) & $0: 46: 26.5$ & $43: 00: 43.0$ & 20.48 & -420.3 & 25.7 & $4 \times 1250$ \\
PN4 (M77) & $0: 43: 32.3$ & $41: 58: 42.5$ & 20.99 & -579.0 & 9.93 & $4 \times 1200$ \\
PN5 (M586) & $0: 43: 07.9$ & $41: 28: 45.5$ & 20.82 & -713.5 & 3.05 & $4 \times 1200$ \\
PN6 (M2750) & $0: 42: 40.3$ & $41: 19: 07.4$ & 21.23 & -766.5 & 0.70 & $4 \times 1200$ \\
PN7 (LAMOST) & $0: 49: 29.0$ & $37: 50: 45.4$ & 21.51 & -203.0 & 50.2 & $4 \times 2400$ \\
& & & & & & $1 \times 1200$ \\
\hline
\end{tabular}

${ }^{a}$ Number in the bracket that follows the PN ID is ID number from Merrett et al. (2006) except PN7, which was newly discovered by LAMOST.

${ }^{b} m(\lambda 5007)=-2.5 \log F(\lambda 5007)-13.74$.

${ }^{c}$ Sky-projected galactocentric distance.

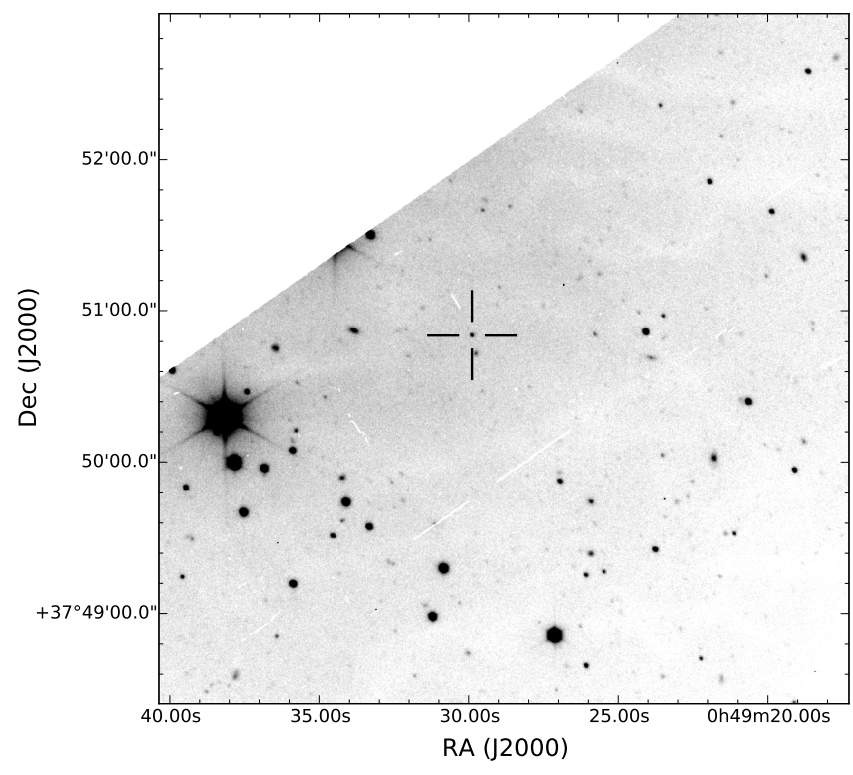

FIG. 2.- Negative greyscale GTC OSIRIS acquisition image of the faint target PN7 (marked with the crosshair). This $g$-band image was taken under photometric condition with seeing $\sim 1^{\prime \prime}$ at an exposure of $30 \mathrm{~s}$.

and suffers from the bright background emission of the galaxy, direct acquisition imaging of all $\mathrm{PNe}$ was made with an exposure of 20-30s. Figure2 is the GTC OSIRIS CCD2 acquisition image of PN7, the faintest target in our sample (Table 1), with an exposure of 30s. The GTC exposures are summarized in Table 1. In order to avoid light losses due to atmospheric diffraction, the long slit was placed along the parallactic angle during exposures. During the observations of each night, exposures of the spectrophotometric standard stars G191-B2B, Ross640 and G158-100 (Oke 1974, 1990) were made. Arc lines of the neon and HgAr lamps were obtained for wavelength calibration. Exposures of the spectrophotometric standards and the arc lamps were made right after observations of each PN target.

\subsection{Data Reduction}

All data were reduced with standard procedures for long-slit spectra using IRAF ${ }^{2}$. The raw two-dimensional (2D) spectra were first combined, and then biassubtracted, flat-fielded, cosmic-ray removed, and wavelength calibrated using exposures of the HgAr lamp. Geometry rectification, which was used to correct for geometry distortion along the slit, was also made during wavelength calibration. The sky background was then fitted along the slit direction using spline functions, and subtracted from the 2D spectra. The 1D spectrum of each target PN was extracted from the background subtracted $2 \mathrm{D}$ spectrum. The $1 \mathrm{D}$ spectra were then corrected for atmospheric extinction, and flux calibrated using observations of the standard stars. As an example, Figure 4 shows the fully calibrated 1D spectra of PN3 and PN7, the brightest and faintest targets in our sample, respectively.

Much effort was made to subtract the sky background. The excellent observing condition at ORM enables detection of the [O III] $\lambda 4363$ auroral line even in the faintest target PN of our sample (Figure 3, top panel). However, the sky background in the red part $(>6000 \AA)$ is relatively strong. Besides, intensity of sky lines is inhomogeneous along the long slit, and this inhomogeneity becomes significant for the strong sky lines such as [O I] $\lambda \lambda 5577,6300,6363$. We used multiple cubic-spline functions to carefully fit the background emission along the slit direction, and then subtract it from the wavelengthcalibrated (also geometry-rectified) 2D spectrum. Subtraction of the background emission is generally satisfactory (e.g., Figure 3. bottom panel) except PN6, which is close to the center of M31 and thus its spectrum is affected by the strong galactic background emission. Despite much effort, the signal-to-noise $(\mathrm{S} / \mathrm{N})$ ratio of the $1 \mathrm{D}$ spectrum of PN6 is lower than those of the other six targets.

The [O III $\lambda 4363$ auroral line is seen in the reduced 2D spectra of all seven PNe (e.g., Figure 3 , bottom panel). The $[\mathrm{N} \mathrm{II}] \lambda 5755$ auroral line is also detected in the spectra of relatively bright $\mathrm{PNe}$. We also managed to discern the nebular emission of the [O I] $\lambda \lambda 6300,6363$ lines in

2 IRAF, the Image Reduction and Analysis Facility, is distributed by the National Optical Astronomy Observatory, which is operated by the Association of Universities for Research in Astronomy under cooperative agreement with the National Science Foundation. 
the spectra of all targets except PN6. The [S III $\lambda 6312$ auroral line, which is close to $[\mathrm{O} \mathrm{I}] \lambda 6300$, was detected in PN1, PN2, PN3 and PN7 (Figure 4). The former three PNe are bright and located in the outskirt Northern Spur, and the latter one in the outer stream of M31, far $(\sim 3.65$; see Figure 1) from the galactic center. These four PNe also suffer much less from the galactic background emission than the other three.

We noticed the presence of second-order contamination in the final 1D spectra of the targets (Figure 4), although the R1000B grism was claimed to be free of such effect. Our derived response curve shows that the second-order contamination is obvious above $6300 \AA$. We corrected the red part (6200-7760 $\AA$ ) of the response curve by carrying out global fitting using a five-order polynomial. The efficiency curve of R1000B given by the OSIRIS User Manual ${ }^{3}$ was used as a template. The final corrected R1000B response curve is safe for flux calibration (A. Cabrera-Lavers, GTC, private communications).

\subsection{Extinction Correction}

The logarithmic extinction parameter at $\mathrm{H} \beta, c(\mathrm{H} \beta)$, was derived for the seven M31 PNe by comparing the observed H I Balmer line ratios with the predicted Case B values. It is worth noting that some low-order $\mathrm{H}$ I Balmer lines are blended with $\mathrm{He}$ II lines: $\mathrm{H} \gamma$ is blended with He II $\lambda 4338\left(4 \mathrm{f}^{2} \mathrm{~F}^{\mathrm{o}}-10 \mathrm{~g}^{2} \mathrm{G}\right), \mathrm{H} \beta$ is blended with He II $\lambda 4859\left(4 \mathrm{f}^{2} \mathrm{~F}^{\mathrm{o}}-8 \mathrm{~g}^{2} \mathrm{G}\right)$, and $\mathrm{H} \alpha$ with He II $\lambda 6560\left(4 \mathrm{f}^{2} \mathrm{~F}^{\mathrm{o}}\right.$ $-6 \mathrm{~g}^{2} \mathrm{G}$ ). Flux contributions of these He II lines are negligible $(<2 \%)$. The other low-order Balmer lines were not used for extinction correction because $\mathrm{H}$ I $\lambda 3889$ ( $n$ $=2-8)$ is blended with the He I $\lambda 3888\left(2 \mathrm{~s}^{3} \mathrm{~S}-3 \mathrm{p}^{3} \mathrm{P}^{\mathrm{o}}\right)$ line, H I $\lambda 3970(n=2-7)$ is blended with [Ne III] $\lambda 3967$ $\left(2 \mathrm{p}^{4}{ }^{3} \mathrm{P}_{1}-{ }^{1} \mathrm{D}_{2}\right)$, and $\mathrm{H} \delta(\lambda 4101)$ is probably blended with the $\mathrm{N}$ III $\lambda \lambda 4097,4103\left(3 \mathrm{~s}^{2} \mathrm{~S}-3 \mathrm{p}^{2} \mathrm{P}^{\mathrm{o}}\right)$ lines, although the flux contribution of the latter is expected to be negligible.

The average $c(\mathrm{H} \beta)$ value derived from the $\mathrm{H} \alpha / \mathrm{H} \beta$ and $\mathrm{H} \gamma / \mathrm{H} \beta$ ratios, as adopted for all $\mathrm{PNe}$, are presented in Table 2. Here the theoretical H I Balmer line ratios were adopted from Storey \& Hummer (1995), assuming an electron temperature of $10000 \mathrm{~K}$ and a density of $10^{4} \mathrm{~cm}^{-3}$. Given that the $\mathrm{H}$ I recombination line ratios are insensitive to the electron temperature and essentially independent on the density, this assumption of $T_{\mathrm{e}}$ and $N_{\mathrm{e}}$ is reasonable. The observed line fluxes were dereddened by

$$
I(\lambda)=10^{c(\mathrm{H} \beta) f(\lambda)} F(\lambda),
$$

where $f(\lambda)$ is the extinction curve adopted from Cardelli et al. (1989) with a total-to-selective extinction ratio $R_{\mathrm{V}}$ $=3.1$. The effects on the extinction-corrected line intensities caused by the use of different reddening curves (e.g., Savage \& Mathis 1979, Cardelli et al. 1989, Clayton et al. 2015) was assessed. They resulted in very small differences in the dereddened intensities of emission lines in the wavelength range $3630-7760 \AA$ covered by the R1000B grism of GTC OSIRIS. The observed fluxes and the extinction-corrected intensities of the emission lines detected in all seven M31 PNe, both normalized to $\mathrm{H} \beta$

3 URL http://www.gtc.iac.es/instruments/osiris/media/OSIRISUSER-MANUAL_v3_1.pdf
$=100$, are presented in Table 2 . The total $\mathrm{H} \beta$ fluxes of the targets, as integrated in the extracted spectra, are also presented. Given that the slit width (1.'0) we used is larger than the typical seeing $\left(00^{\prime \prime} 6-0{ }^{\prime \prime} 8\right)$ and that the slit is always placed along the parallactic angle during exposures, no significant light loss in $\mathrm{H} \beta$ is expected.

\section{RESULTS}

\subsection{Relative Line Intensities}

We present in Table 2 the normalized (to $\mathrm{H} \beta=100$ ) intensities and measurement errors of the emission lines. For brighter PNe, the typical uncertainties in fluxes of the [O III] $\lambda 4363$ auroral line is less than $10 \%$. For the PN1, PN2 and PN3, the relatively bright targets in our sample, the $\mathrm{S} / \mathrm{N}$ ratios of the $\lambda 4363$ line are $\sim 30$, 21 and $>50$, respectively. For the faintest target PN7, $\mathrm{S} / \mathrm{N}\left(\left[\begin{array}{l}\mathrm{O} \\ \mathrm{III}\end{array}\right] \lambda 4363\right) \sim 10$. Electron temperature diagnostics using the $[\mathrm{O}$ III $](\lambda 4959+\lambda 5007) / \lambda 4363$ line ratio are presented in Section 3.3 .

Some emission lines detected in our spectra have blending issues. We corrected the flux of the [Ne III] $\lambda 3967$ line for the blended H I $\lambda 3970$ line, using the theoretical hydrogen line ratio of Storey \& Hummer (1995). However, for all targets, the corrected [Ne III $\lambda 33967$ (dereddened) line flux still yields higher $\mathrm{Ne}^{2+} / \mathrm{H}^{+}$abundance ratios than that derived from [Ne III] $\lambda 3868$, which is free of line blending. The [Ar IV] $\lambda 4711$ line is blended with He I $\lambda 4713\left(2 \mathrm{p}^{3} \mathrm{P}^{\mathrm{o}}-4 \mathrm{~s}^{3} \mathrm{~S}\right)$, which contributes $30-40 \%$ to the total flux of the [Ar IV] line for all seven targets. The He I line flux was estimated using the theoretical Case B He I $\lambda 4713 / \lambda 4471$ ratio calculated by Porter et al. (2012), whose calculation for this ratio only differs from that of Benjamin et al. (1999) by less than 0.5\%. The [S III] $\lambda 6312$ line is blended with He II $\lambda 6311\left(5 \mathrm{~g}^{2} \mathrm{G}-\right.$ $\left.16 \mathrm{~h}^{2} \mathrm{H}^{\circ}\right)$, which typically contributes less than $1 \%$ to the total flux. For PN3, whose He II $\lambda 4686$ is the strongest among the targets, flux contribution of the He II $\lambda 6311$ line is close to $5 \%$.

Some permitted lines of heavy elements were detected (Table 2). These lines are faint, with a typical intensity $<5 \%$ of $\mathrm{H} \beta$. The $\mathrm{N}$ III $\lambda 4641\left(\mathrm{M} 23 \mathrm{p}^{2} \mathrm{P}_{3 / 2}^{\mathrm{o}}-3 \mathrm{~d}^{2} \mathrm{D}_{5 / 2}\right)$ line was well detected in the spectrum of PN3 (Figure 4). This line is blended with N III $\lambda 4634,4642$ of the same multiplet, and is probably blended with the O II M1 $3 \mathrm{~s}^{4} \mathrm{P}-3 \mathrm{p}^{4} \mathrm{D}^{\circ}$ lines (see for example, Figure 1 in Liu et al. 1995 or Figure 32 in Fang \& Liu 2013). The dominant excitation mechanism of the $[\mathrm{N}$ III $] \lambda 4641$ line is the starlight and/or Bowen fluorescence (e.g., Bowen 1935 Grandi 1976). Thus this line is unsuitable for the ionic abundance $\left(\mathrm{N}^{3+} / \mathrm{H}^{+}\right)$calculation. Carbon emission lines were detected in the spectrum of PN2, as discussed in Section 3.2 below.

The uncertainties of the emission line intensities were derived by adding quadratically the uncertainties associated with the Poisson noise of the line and the CCD and background noise. The former scales with the electron counts of the line, and the latter scales with the local noise and the full line width. The error contributions from systematic effects, such as flux calibration, subtraction of the background level, determination of reddening, or line blending, are expected to be much smaller and were not taken into account. Our flux calibration is reliable as the extinction parameter $c(\mathrm{H} \beta)$ derived from 

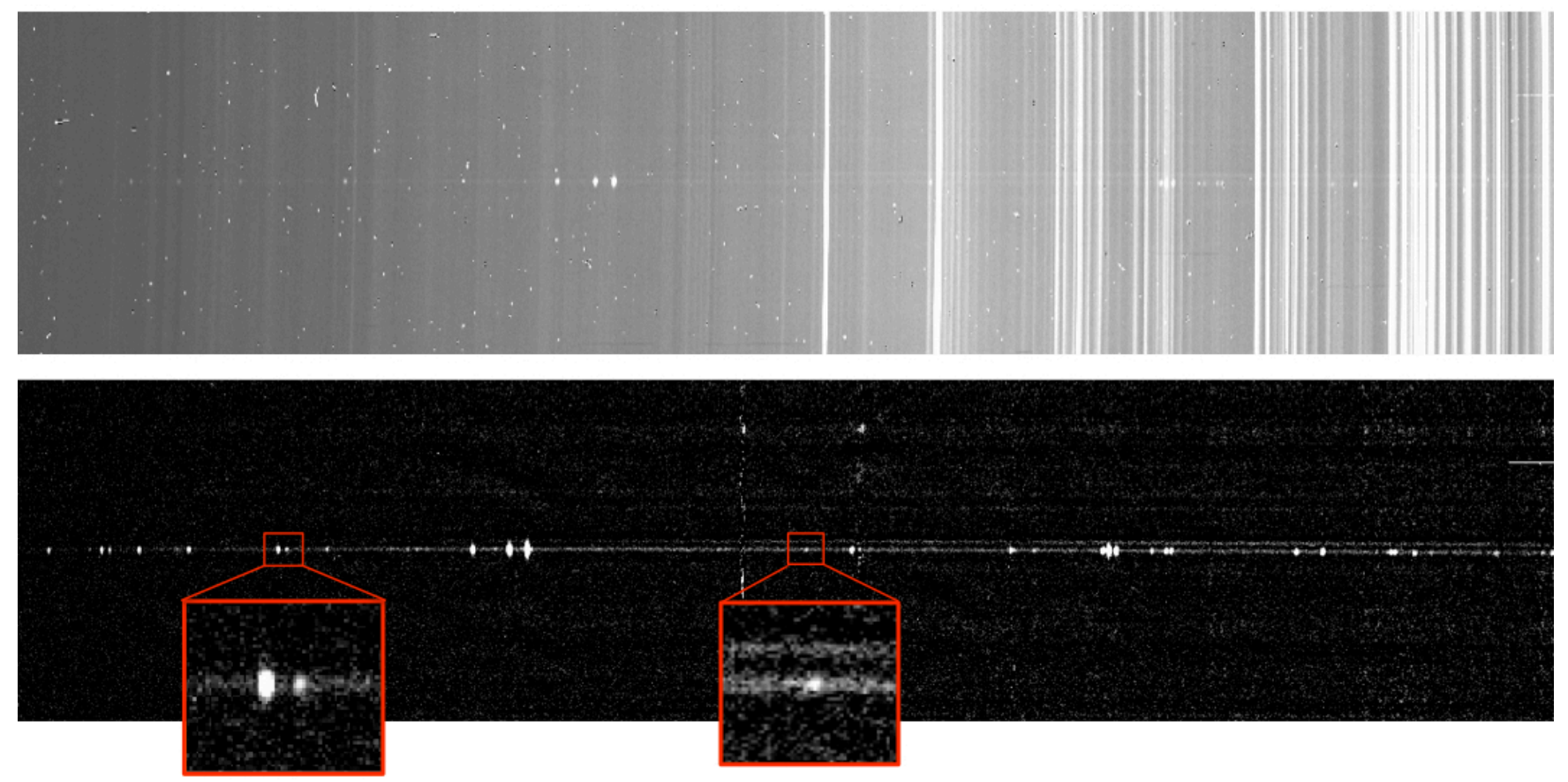

FIG. 3.- GTC OSIRIS 2D spectrum of PN7, the faintest target in our sample. The top image is the raw data and the bottom image shows the spectrum after cosmic-ray removal, wavelength calibration, geometry rectification, and background subtraction. Images have been trimmed to a size of $450 \times 2000$ pixels. Please note the shift of emission line positions (along the dispersion direction) on CCD after geometry rectification and wavelength calibration. Wavelength coverage is 3630-7750 A. The two images, both presented in logarithm, are scaled to different levels. The insets of the bottom image show the $\mathrm{H} \gamma$ and [O III] $\lambda 4363$ lines (left) and the [N II] $\lambda 5755$ line (right).

the $\mathrm{H} \gamma / \mathrm{H} \beta$ ratio generally agrees with that derived from $\mathrm{H} \alpha / \mathrm{H} \beta$. The important nebular emission lines of our $\mathrm{PNe}$ are generally not much affected by the sky lines (e.g., the mercury line $\mathrm{Hg}$ I $\lambda 4358$ is faint at ORM, La Palma, and the detection of the [O III $\lambda 4363$ auroral is not much affected). Moreover, the background subtraction in the 2D spectra seemed flawless (e.g., Figure 3 see also the description of data reduction in Section 2.3). The errors introduced by reddening are also small, less than $5 \%$ as discussed in Section 2.4. Finally, line blending was also considered when analyzing the emission lines. Fortunately, errors due to this effect are mostly negligible for the lines that are critical for plasma diagnostics and abundance determinations (Table 2), except for the [Ar IV] $\lambda 4711$ line.

\subsection{Carbon Emission Lines}

Very broad C IV $\lambda 5805\left(\mathrm{M} 13 \mathrm{~s}^{2} \mathrm{~S}-3 \mathrm{p}^{2} \mathrm{P}^{\mathrm{o}}\right)$ line, with FWHM $\sim 43 \AA$, was detected in the spectrum of PN2 (Figure 5 right). This line is a blend of two fine-structure components $\lambda \lambda 5801.33,5811.98^{4}$, and can be attributed to the emission of a Wolf-Rayet (WR) type central star. The same feature has been detected in the spectra of another two PNe in the outer disk of M31 by Balick et al. (2013, Figure 2). The extinction-corrected flux of the C IV $\lambda 5805$ line in our PN2 is $1.20 \times 10^{-16} \mathrm{erg} \mathrm{cm}^{-2} \mathrm{~s}^{-1}$, with an uncertainty of $\sim 10 \%$. This flux is generally in line with those observed by Balick et al. (2013).

Also detected in the spectrum of $\mathrm{PN} 2$ are the $\mathrm{C}$ III $\lambda 4665\left(\mathrm{M} 53 \mathrm{~s}^{\prime}{ }^{3} \mathrm{P}_{2}^{\mathrm{o}}-3 \mathrm{p}^{\prime 3} \mathrm{P}_{2}\right)$ and $\mathrm{C}$ II $\lambda 4267\left(\mathrm{M} 63 \mathrm{~d}^{2} \mathrm{D}-\right.$

4 The wavelengths of these two C IV lines are adopted from the laboratory wavelengths in the atomic spectral line lists compiled by Hirata \& Horaguchi 1995
$4 \mathrm{f}^{2} \mathrm{~F}^{\circ}$ ) lines. The high-excitation $\mathrm{C}$ III $\lambda 4665$ line might be due to emission of the WR central star. A Gaussian profile with FWHM $\sim 11 \AA$ seems to well fit this line (Figure 5. middle), but its accurate profile is difficult to discern due to noise and line blending. C III $\lambda 4665$ is probably blended with the $\mathrm{C}$ IV $\lambda 4658$ (M8 $5 \mathrm{~g}^{2} \mathrm{G}-$ $6 \mathrm{~h}^{2} \mathrm{H}^{\mathrm{o}}$ ) and $\mathrm{C}$ III $\lambda 4649\left(\mathrm{M} 13 \mathrm{~s}^{3} \mathrm{~S}-3 \mathrm{p}^{3} \mathrm{P}^{\mathrm{o}}\right)$ recombination lines.

The C II $\lambda 4267$ optical recombination line (ORL) is produced by radiative recombination only, and thus is due to nebular emission. Its line width, although difficult to discern due to noise and possible blending (Figure 5. left), seems narrower than that of the C IV $\lambda 5805$ line. The extinction-corrected flux of the $\lambda 4267$ line is $\sim 2.1 \times 10^{-17} \mathrm{erg} \mathrm{cm}^{-2} \mathrm{~s}^{-1}$, with an uncertainty over $50 \%$. This flux value is close to that observed in one of the two PNe of Balick et al. (2013), which is $\sim 1.8 \times 10^{-17}$ $\operatorname{erg~cm} \mathrm{cm}^{-2} \mathrm{~s}^{-1}$. The C II $\lambda 4267$ line was used to calculate the $\mathrm{C}^{2+} / \mathrm{H}^{+}$abundance ratio in Section 3.4 .

\subsection{Plasma Diagnostics}

Plasma diagnostics were determined using the collisionally excited lines ${ }^{5}$ (CELs) of heavy elements in conventional manner (e.g., Osterbrock \& Ferland 2006). The [O III $](\lambda 4959+\lambda 5007) / \lambda 4363$ auroral-to-nebular line ratio was used to derive the electron temperature. The [N II] $\lambda 5755$ auroral line, whose flux is typically $\sim 5-15 \%$ of that of the [O III $\lambda 4363$ line, was detected in all targets except PN6, and the $[\mathrm{N} \mathrm{II}](\lambda 6548+\lambda 6583) / \lambda 5755$ ratio was also used as a temperature diagnostic. The [Ar IV] $\lambda 4711 / \lambda 4740$ and $[\mathrm{S} \mathrm{II}] \lambda 6716 / \lambda 6731$ nebular line ratios

\footnotetext{
5 Quite often, those lines are electron dipole-forbidden transitions, so they are also commonly called forbidden lines.
} 

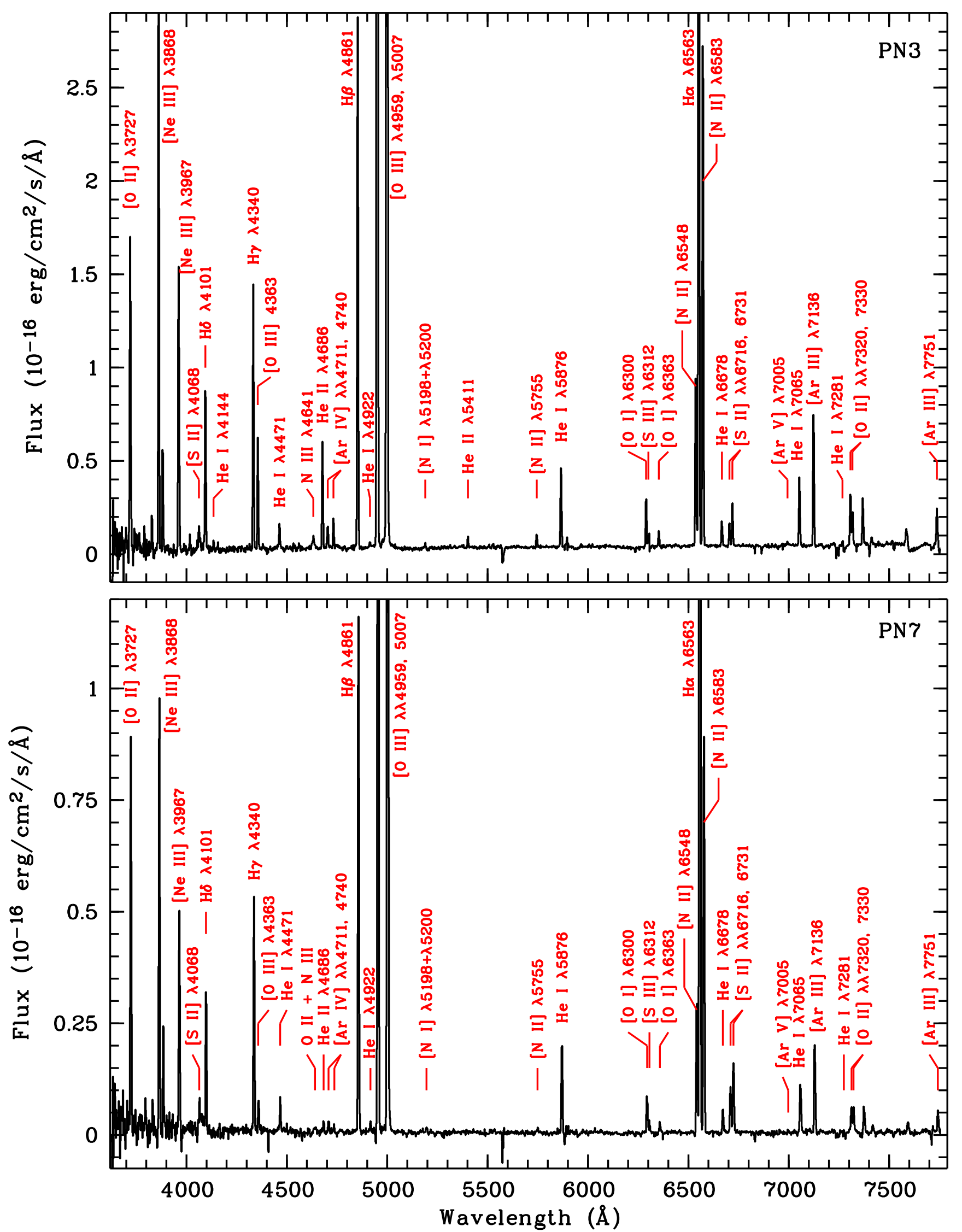

FIG. 4.- GTC OSIRIS 1D spectrum of PN3 (top) and PN7 (bottom), the brightest and the faintest targets in our sample (Table 1) respectively. All important emission lines are labeled. Extinction has not been corrected for. The features between the [O II] $\lambda \lambda 7320,7330$ and [Ar III] $\lambda 7751$ lines are the second-order contamination, probably belong to [Ne III] $\lambda 3868, \mathrm{H}$ I $\lambda 3889$ and [Ne III] $\lambda 3967$. 
TABLE 2

FLUXes AND INTENSities $^{a}$

\begin{tabular}{|c|c|c|c|c|c|c|c|c|c|c|}
\hline \multirow[t]{2}{*}{ Ion } & \multirow{2}{*}{$\begin{array}{l}\lambda \\
(\AA)\end{array}$} & \multirow[t]{2}{*}{ Transition } & \multicolumn{2}{|r|}{$\mathrm{PN} 1$} & \multicolumn{2}{|r|}{$\mathrm{PN} 2$} & \multicolumn{2}{|r|}{$\mathrm{PN} 3$} & \multicolumn{2}{|r|}{ PN4 } \\
\hline & & & $F(\lambda)$ & $I(\lambda)$ & $F(\lambda)$ & $I(\lambda)$ & $F(\lambda)$ & $I(\lambda)$ & $F(\lambda)$ & $I(\lambda)$ \\
\hline$[\mathrm{O} \quad \mathrm{II}]$ & $3727^{b}$ & $2 \mathrm{p}^{3}{ }^{4} \mathrm{~S}^{\mathrm{o}}-2 \mathrm{p}^{32} \mathrm{D}^{\mathrm{o}}$ & 47.2 & $54.6 \pm 5.4$ & 79.3 & $91.8 \pm 9.1$ & 58.2 & $66.4 \pm 6.6$ & 56.2 & $67.6 \pm 6.7$ \\
\hline $\mathrm{H} \mathrm{I}$ & 3798 & $2 \mathrm{p}^{2} \mathrm{P}^{\mathrm{o}}-10 \mathrm{~d}^{2} \mathrm{D}$ & 4.28 & $4.92 \pm 1.35$ & 8.42 & $9.69 \pm 2.60$ & 3.01 & $3.42 \pm 0.94$ & 4.51 & $5.38 \pm 1.48$ \\
\hline $\mathrm{H}$ I & 3835 & $2 \mathrm{p}^{2} \mathrm{P}^{\mathrm{o}}-9 \mathrm{~d}^{2} \mathrm{D}$ & 5.84 & $6.68 \pm 1.70$ & 6.06 & $6.94 \pm 1.74$ & 4.88 & $5.52 \pm 1.38$ & 3.22 & $3.82 \pm 1.00$ \\
\hline [Ne III] & 3868 & $2 \mathrm{p}^{4}{ }^{3} \mathrm{P}_{2}-2 \mathrm{p}^{41} \mathrm{D}_{2}$ & 74.4 & $84.8 \pm 4.2$ & 112 & $128 \pm 6$ & 104 & $117 \pm 6$ & 69.1 & $81.7 \pm 4.0$ \\
\hline $\mathrm{H} \mathrm{I}_{\mathrm{I}}$ & $3889^{c}$ & $2 \mathrm{p}^{2} \mathrm{P}^{\mathrm{o}}-8 \mathrm{~d}^{2} \mathrm{D}$ & 16.2 & $18.4 \pm 2.0$ & 16.0 & $18.2 \pm 2.0$ & 16.8 & $19.0 \pm 2.0$ & 14.5 & $17.1 \pm 1.9$ \\
\hline [Ne III $]$ & $3967^{d}$ & $2 \mathrm{p}^{43} \mathrm{P}_{1}-2 \mathrm{p}^{41} \mathrm{D}_{2}$ & 41.2 & $46.5 \pm 3.6$ & 52.3 & $59.1 \pm 4.6$ & 53.8 & $60.0 \pm 4.6$ & 38.3 & $44.6 \pm 3.5$ \\
\hline He I & 4026 & $2 \mathrm{p}^{3} \mathrm{P}^{\mathrm{o}}-5 \mathrm{~d}^{3} \mathrm{D}$ & 2.21 & $2.47 \pm 0.50$ & 3.57 & $4.00 \pm 0.83$ & 1.89 & $2.10 \pm 0.42$ & 2.57 & $2.97 \pm 0.60$ \\
\hline$[\mathrm{S}$ II $]$ & $4068^{e}$ & $3 \mathrm{p}^{3}{ }^{4} \mathrm{~S}_{3 / 2}^{\mathrm{o}}-3 \mathrm{p}^{3}{ }^{2} \mathrm{P}_{3 / 2}^{\mathrm{o}}$ & 4.56 & $5.10 \pm 1.78$ & 7.78 & $8.67 \pm 2.20$ & 5.99 & $6.60 \pm 1.60$ & 3.13 & $3.60 \pm 1.10$ \\
\hline $\mathrm{H}$ I & 4101 & $2 \mathrm{p}^{2} \mathrm{P}^{\mathrm{o}}-6 \mathrm{~d}^{2} \mathrm{D}$ & 26.8 & $30.0 \pm 3.3$ & 29.1 & $32.4 \pm 3.6$ & 29.5 & $32.4 \pm 3.6$ & 28.9 & $33.0 \pm 3.6$ \\
\hline He I & 4144 & $2 p^{1} P_{1}^{o}-6 d^{1} D_{2}$ & 2.24 & $2.50 \pm 0.74$ & $\ldots$ & $\ldots$ & 1.15 & $1.26 \pm 0.38$ & $\ldots$ & $\ldots$ \\
\hline $\mathrm{C}_{\text {II }}$ & 4267 & $3 d^{2} D-4 f^{2} F^{o}$ & $\ldots$ & $\ldots$ & 1.60 & $1.74 \pm 0.43$ & $\ldots$ & $\ldots$ & $\ldots$ & $\ldots$ \\
\hline $\mathrm{H}$ I & $4340^{f}$ & $2 \mathrm{p}^{2} \mathrm{P}^{\mathrm{o}}-5 \mathrm{~d}^{2} \mathrm{D}$ & 44.4 & $47.7 \pm$ & & & 43.6 & $46.5 \pm 2.0$ & 42.3 & $46.3 \pm 2.0$ \\
\hline [O III $]$ & 4363 & $2 \mathrm{p}^{2}{ }^{1} \mathrm{D}_{2}-2 \mathrm{p}^{2}{ }^{1} \mathrm{~S}_{0}$ & 8.28 & & & 12.8 & 18.1 & $19.2 \pm$ & 8.63 & $9.42 \pm 1.13$ \\
\hline $\mathrm{He} \mathrm{I}$ & 4471 & $2 \mathrm{p}^{3} \mathrm{P}^{\mathrm{o}}-4 \mathrm{~d}^{3} \mathrm{D}$ & 5.58 & $5.88 \pm$ & 6.18 & 6.52 & 5.10 & $5.35 \pm 0.85$ & 6.11 & $6.53 \pm 1.05$ \\
\hline $\mathrm{N}$ III & $4641^{g}$ & $3 \mathrm{p}^{2} \mathrm{P}_{3 / 2}^{\mathrm{o}}-3 \mathrm{~d}^{2} \mathrm{D}_{5 / 2}$ & 0.83 & $0.9 \pm 0.5$ & 2.03 & $2.09 \pm 0.52$ & 3.57 & $3.67 \pm 0.90$ & $\ldots$ & $\ldots$ \\
\hline C III & $4665^{h}$ & $3 \mathrm{~s}^{\prime 3} \mathrm{P}_{2}^{\mathrm{o}}-3 \mathrm{p}^{\prime 3} \mathrm{P}_{2}$ & $\ldots$ & $\ldots$ & 2.77 & $2.84 \pm 0.71$ & & - & $\ldots$ & \\
\hline He II & 4686 & $3 \mathrm{~d}^{2} \mathrm{D}-4 \mathrm{f}^{2} \mathrm{~F}^{\mathrm{o}}$ & 0.74 & $0.76 \pm 0.30$ & 4.00 & $4.10 \pm 0.60$ & 18.6 & $19.0 \pm 1.7$ & 1.36 & $1.40 \pm 0.43$ \\
\hline$[$ Ar IV $]$ & $4711^{i}$ & $3 p^{3}{ }^{4} S_{3 / 2}^{o}-3 p^{3}{ }^{2} D_{5 / 2}^{o}$ & 1.13 & $1.15 \pm 0.35$ & 1.21 & $1.24 \pm 0.20$ & 4.41 & $4.49 \pm 0.67$ & 2.18 & $2.24 \pm 0.68$ \\
\hline$[$ Ar IV] & 4740 & $3 \mathrm{p}^{3}{ }^{4} \mathrm{~S}_{3 / 2}^{\mathrm{o}}-3 \mathrm{p}^{32} \mathrm{D}_{3 / 2}^{\mathrm{o}}$ & 1.03 & $1.04 \pm 0.34$ & 1.01 & $1.02 \pm 0.20$ & 5.19 & $5.27 \pm 0.80$ & 1.97 & $2.01 \pm 0.60$ \\
\hline $\mathrm{H}$ I & 4861 & $2 \mathrm{p}^{2} \mathrm{P}^{\mathrm{o}}-4 \mathrm{~d}^{2} \mathrm{D}$ & 100 & 100 & 100 & 100 & 100 & 100 & 100 & 100 \\
\hline He I & 4922 & $2 \mathrm{p}^{1} \mathrm{P}_{1}^{\mathrm{o}}-4 \mathrm{~d}^{1} \mathrm{D}_{2}$ & 2.0 & $2.0 \pm 0.8$ & 1.97 & $1.96 \pm 0.77$ & 1.20 & $1.20 \pm 0.47$ & 1.90 & $1.89 \pm 0.75$ \\
\hline$[\mathrm{O} \mathrm{III}]$ & 4959 & $2 \mathrm{p}^{2}{ }^{1} \mathrm{P}_{1}-2 \mathrm{p}^{21} \mathrm{D}_{2}$ & 345 & $342 \pm 17$ & 434 & $429 \pm 21$ & 482 & $478 \pm 23$ & 321 & $317 \pm 15$ \\
\hline$[\mathrm{O}$ III] & 5007 & $2 \mathrm{p}^{2}{ }^{1} \mathrm{P}_{2}-2 \mathrm{p}^{2}{ }^{1} \mathrm{D}_{2}$ & 1042 & $1025 \pm 31$ & 1297 & $1275 \pm 38$ & 1414 & $1393 \pm 42$ & 956 & $937 \pm 28$ \\
\hline$[\mathrm{N}$ I $]$ & $5198^{j}$ & $2 \mathrm{p}^{3}{ }^{4} \mathrm{~S}_{3 / 2}^{\mathrm{o}}-2 \mathrm{p}^{3}{ }^{2} \mathrm{D}_{3 / 2}^{\mathrm{o}}$ & $\ldots$ & $\ldots$ & 1.80 & $1.73 \pm 0.50$ & 0.67 & $0.65 \pm 0.21$ & $\ldots$ & $\ldots$ \\
\hline He II & 5411 & $4 \mathrm{f}^{2} \mathrm{~F}^{\mathrm{o}}-7 \mathrm{~g}^{2} \mathrm{G}$ & $\ldots$ & $\ldots$ & $\ldots$ & $\ldots$ & 1.81 & $1.72 \pm 0.34$ & $\ldots$ & $\cdots$ \\
\hline$[\mathrm{Cl} \mathrm{III}]$ & 5517 & $3 p^{3}{ }^{4} S_{3 / 2}^{o}-3 p^{3}{ }^{2} D_{5 / 2}^{o}$ & $\ldots$ & $\ldots$ & $\ldots$ & $\ldots$ & 0.48 & $0.45 \pm 0.20$ & $\ldots$ & $\ldots$ \\
\hline$[\mathrm{Cl} \mathrm{III}]$ & 5537 & $3 \mathrm{p}^{3}{ }^{4} \mathrm{~S}_{3 / 2}^{\mathrm{o}}-3 \mathrm{p}^{32} \mathrm{D}_{3 / 2}^{\mathrm{o}}$ & $\ldots$ & $\ldots$ & $\ldots$ & $\ldots$ & 0.72 & $0.68 \pm 0.21$ & $\ldots$ & $\ldots$ \\
\hline$[\mathrm{N}$ III $]$ & 5755 & $2 \mathrm{p}^{2}{ }^{1} \mathrm{D}_{2}-2 \mathrm{p}^{2}{ }^{1} \mathrm{~S}_{0}$ & 1.90 & $1.76 \pm 0.50$ & 2.06 & $1.89 \pm 0.60$ & 2.64 & $2.45 \pm 0.50$ & 1.53 & $1.38 \pm 0.42$ \\
\hline $\mathrm{C}$ IV & 5805 & $3 \mathrm{~s}^{2} \mathrm{~S}-3 \mathrm{p}^{2} \mathrm{P}^{\mathrm{o}}$ & $\ldots$ & $\ldots$ & & & $\ldots$ & $\ldots$ & $\ldots$ & $\ldots$ \\
\hline He I & 5876 & $2 p^{3} P^{o}-3 d^{3} D$ & 19.1 & $17.4 \pm$ & 2 & 18. & 16.3 & $15.0 \pm 1.6$ & 17.5 & $15.6 \pm 1.7$ \\
\hline$[\mathrm{O}$ I $]$ & 6300 & $2 \mathrm{p}^{4}{ }^{3} \mathrm{P}_{2}-2 \mathrm{p}^{4}{ }^{1} \mathrm{D}_{2}$ & 4.10 & $3.64 \pm 0.94$ & 10 & $9.60 \pm 2.40$ & 8.82 & $7.93 \pm 2.00$ & 5.42 & $4.68 \pm 1.20$ \\
\hline [S III] & $6312^{k}$ & $3 \mathrm{p}^{2}{ }^{1} \mathrm{D}_{2}-3 \mathrm{p}^{2}{ }^{1} \mathrm{~S}_{0}$ & 1.37 & $1.21 \pm 0$ & 2. & $2.26 \pm 0.69$ & 1.41 & $1.26 \pm 0.25$ & $\ldots$ & $\ldots$ \\
\hline$\left[\begin{array}{ll}\mathrm{O} & \mathrm{I}\end{array}\right]$ & 6363 & $2 \mathrm{p}^{4}{ }^{3} \mathrm{P}_{1}-2 \mathrm{p}^{4}{ }^{1} \mathrm{D}_{2}$ & 1.8 & $1.63 \pm 0$ & 4. & $4.00 \pm 1$ & 3.32 & $2.97 \pm 1.33$ & 2.30 & $1.98 \pm 0.88$ \\
\hline$\left[\begin{array}{lll}\mathrm{N} & \mathrm{II}\end{array}\right]$ & 6548 & $2 \mathrm{p}^{2}{ }^{3} \mathrm{P}_{1}-2 \mathrm{p}^{2}{ }^{1} \mathrm{D}_{2}$ & 21 & $18.4 \pm$ & 38.0 & $33.3 \pm$ & 34.0 & $30.2 \pm 2.3$ & 22.6 & $19.1 \pm 2.3$ \\
\hline $\mathrm{H}$ I & 6563 & $2 \mathrm{p}^{2} \mathrm{P}^{\mathrm{o}}-2 \mathrm{~d}^{2} \mathrm{D}$ & 33 & $293=$ & 356 & 310 & 328 & $291 \pm 1$ & 341 & $288 \pm 1$ \\
\hline$[\mathrm{N}$ II $]$ & 6583 & $2 \mathrm{p}^{2}{ }^{3} \mathrm{P}_{2}-2 \mathrm{p}^{2}{ }^{1} \mathrm{D}_{2}$ & 64 & $56.5 \pm 6.4$ & 1 & $97.9 \pm$ & 99.6 & $88.2 \pm$ & 68.2 & $57.5 \pm 6.3$ \\
\hline He I & $6678^{l}$ & $2 \mathrm{p}^{1} \mathrm{P}_{1}^{\mathrm{o}}-3 \mathrm{~d}^{1} \mathrm{D}_{2}$ & 5.76 & $5.01 \pm 0.75$ & 6.88 & $5.97 \pm$ & 4.74 & $4.17 \pm$ & 5.46 & $4.58 \pm 0.68$ \\
\hline [S II $]$ & 6716 & $3 \mathrm{p}^{3}{ }^{4} \mathrm{~S}_{3 / 2}^{\mathrm{o}}-3 \mathrm{p}^{3}{ }^{2} \mathrm{D}_{5 / 2}^{\mathrm{o}}$ & 2.17 & $1.88 \pm 0.20$ & 5.21 & $4.51 \pm 0.50$ & 4.34 & $3.82 \pm 0.43$ & 7.24 & $6.05 \pm 0.67$ \\
\hline$[\mathrm{S}$ II $]$ & 6731 & $3 p^{3}{ }^{4} \mathrm{~S}_{3 / 2}^{\mathrm{o}}-3 \mathrm{p}^{3}{ }^{2} \mathrm{D}_{3 / 2}^{\mathrm{o}}$ & 4.00 & $3.45 \pm 0.42$ & 9.24 & $8.00 \pm 1.01$ & 8.39 & $7.37 \pm 0.90$ & 9.97 & $8.32 \pm 0.92$ \\
\hline$[\mathrm{Ar} \mathrm{V}]$ & 7005 & $3 \mathrm{p}^{2}{ }^{3} \mathrm{P}_{2}-3 \mathrm{p}^{2}{ }^{1} \mathrm{D}_{2}$ & $\ldots$ & $\ldots$ & 0.70 & $0.60 \pm 0.12$ & 0.77 & $0.67 \pm 0.13$ & $\ldots$ & $\ldots$ \\
\hline He I & 7065 & $2 \mathrm{p}^{3} \mathrm{P}^{\mathrm{o}}-3 \mathrm{~s}^{3} \mathrm{~S}$ & 13.9 & $11.8 \pm 1.5$ & 15.7 & $13.3 \pm 1.7$ & 13.2 & $11.3 \pm 1.4$ & 9.24 & $7.52 \pm 1.00$ \\
\hline [Ar III] & 7136 & $3 p^{4}{ }^{3} P_{2}-3 p^{4}{ }^{1} D_{2}$ & 18.4 & $15.5 \pm 2.0$ & 19.1 & $16.1 \pm 2.1$ & 24.7 & $21.2 \pm 2.7$ & 18.8 & $15.2 \pm 2.0$ \\
\hline He I & 7281 & $2 \mathrm{p}^{1} \mathrm{P}_{1}^{\mathrm{o}}-3 \mathrm{~s}^{1} \mathrm{~S}_{0}$ & 0.55 & $0.46 \pm 0.12$ & $\ldots$ & $\ldots$ & 0.62 & $0.52 \pm 0.13$ & 0.55 & $0.44 \pm 0.11$ \\
\hline$\left[\begin{array}{lll}\mathrm{O} & \mathrm{II}\end{array}\right]$ & 7320 & $2 \mathrm{p}^{3}{ }^{2} \mathrm{D}_{5 / 2}^{\mathrm{o}}-2 \mathrm{p}^{3}{ }^{2} \mathrm{P}_{3 / 2}^{\mathrm{o}}$ & 8.40 & $7.02 \pm 1.14$ & 9.79 & $8.17 \pm 1.22$ & 10.4 & $8.82 \pm 1.32$ & 5.62 & $4.49 \pm 0.67$ \\
\hline$\left[\begin{array}{ll}\mathrm{O} & \mathrm{II}\end{array}\right]$ & $7330^{m}$ & $2 \mathrm{p}^{32} \mathrm{D}_{3 / 2}^{\mathrm{o}}-2 \mathrm{p}^{32} \mathrm{P}_{3 / 2}^{\mathrm{o}}$ & 8.08 & $6.74 \pm 1.00$ & 10.9 & $9.13 \pm 1.37$ & 7.13 & $6.06 \pm 0.90$ & 4.21 & $3.36 \pm 0.50$ \\
\hline [Ar III] & 7751 & $3 \mathrm{p}^{4}{ }^{3} \mathrm{P}_{1}-3 \mathrm{p}^{4}{ }^{1} \mathrm{D}_{2}$ & 3.86 & $3.14 \pm 1.16$ & 1.67 & $1.35 \pm 0.47$ & 7.28 & $6.05 \pm 2.45$ & 5.31 & $4.10 \pm 1.44$ \\
\hline \multicolumn{3}{|l|}{$c(\mathrm{H} \beta)$} & \multicolumn{2}{|r|}{0.196} & \multicolumn{2}{|r|}{0.198} & \multicolumn{2}{|r|}{0.177} & \multicolumn{2}{|r|}{0.247} \\
\hline \multicolumn{2}{|c|}{$\log F(\mathrm{H} \beta)^{n}$} & & \multicolumn{2}{|r|}{-14.70} & \multicolumn{2}{|r|}{-14.96} & \multicolumn{2}{|r|}{-14.73} & \multicolumn{2}{|r|}{-14.88} \\
\hline
\end{tabular}

\begin{tabular}{|c|c|c|c|c|c|c|c|c|}
\hline \multirow[t]{2}{*}{ Ion } & \multirow{2}{*}{$\begin{array}{l}\lambda \\
(\AA)\end{array}$} & \multirow[t]{2}{*}{ Transition } & \multicolumn{2}{|c|}{ PN5 } & \multicolumn{2}{|c|}{ PN6 } & \multicolumn{2}{|c|}{ PN7 } \\
\hline & & & $\overline{F(\lambda)}$ & $I(\lambda)$ & $\overline{F(\lambda)}$ & $I(\lambda)$ & $\overline{F(\lambda)}$ & $I(\lambda)$ \\
\hline$\left[\begin{array}{ll}\mathrm{O} & \mathrm{II}\end{array}\right]$ & $3727^{b}$ & $2 \mathrm{p}^{3}{ }^{4} \mathrm{~S}^{\mathrm{o}}-2 \mathrm{p}^{3}{ }^{2} \mathrm{D}^{\mathrm{o}}$ & 42.4 & $59.4 \pm 7.1$ & 33.2 & $46.4 \pm 9.3$ & 73.4 & $84.3 \pm 7.6$ \\
\hline $\mathrm{H}$ I & 3798 & $2 \mathrm{p}^{2} \mathrm{P}^{\mathrm{o}}-10 \mathrm{~d}^{2} \mathrm{D}$ & $\ldots$ & $\ldots$ & & $\ldots$ & 5.23 & $5.97 \pm 1.64$ \\
\hline $\mathrm{H} \mathrm{I}$ & 3835 & $2 \mathrm{p}^{2} \mathrm{P}^{\mathrm{o}}-9 \mathrm{~d}^{2} \mathrm{D}$ & $\ldots$ & & 10.2 & $13.9 \pm 3.5$ & 4.17 & $4.74 \pm 1.20$ \\
\hline$[\mathrm{Ne} \mathrm{III}]$ & 3868 & $2 \mathrm{p}^{4}{ }^{3} \mathrm{P}_{2}-2 \mathrm{p}^{4}{ }^{1} \mathrm{D}_{2}$ & 87.0 & $118 \pm 6$ & 63.0 & $85.4 \pm 4.2$ & 75.6 & $85.6 \pm 4.2$ \\
\hline $\mathrm{H}$ I & $3889^{c}$ & $2 \mathrm{p}^{2} \mathrm{P}^{\mathrm{o}}-8 \mathrm{~d}^{2} \mathrm{D}$ & 13.0 & $17.6 \pm 2.0$ & 16.0 & $21.6 \pm 2.3$ & 18.3 & $20.8 \pm 2.3$ \\
\hline
\end{tabular}


TABLE 2

(CONTINUED)

\begin{tabular}{|c|c|c|c|c|c|c|c|c|}
\hline \multirow[t]{2}{*}{ Ion } & \multirow{2}{*}{$\begin{array}{l}\lambda \\
(\AA)\end{array}$} & \multirow[t]{2}{*}{ Transition } & \multicolumn{2}{|r|}{ PN5 } & \multicolumn{2}{|r|}{ PN6 } & \multicolumn{2}{|r|}{ PN7 } \\
\hline & & & $F(\lambda)$ & $I(\lambda)$ & $F(\lambda)$ & $I(\lambda)$ & $F(\lambda)$ & $I(\lambda)$ \\
\hline$[\mathrm{Ne}$ III] & $3967^{d}$ & $2 \mathrm{p}^{43} \mathrm{P}_{1}-2 \mathrm{p}^{4}{ }^{1} \mathrm{D}_{2}$ & 43.2 & $57.0 \pm 5.0$ & 32.3 & $42.7 \pm 3.3$ & 42.2 & $47.3 \pm 3.7$ \\
\hline$\left[\begin{array}{lll}\mathrm{S} & \mathrm{II}\end{array}\right]$ & $4068^{e}$ & $3 \mathrm{p}^{3}{ }^{4} \mathrm{~S}_{3 / 2}^{\mathrm{o}}-3 \mathrm{p}^{3}{ }^{2} \mathrm{P}_{3 / 2}^{\mathrm{o}}$ & 6.36 & $8.2 \pm 3.2$ & $\ldots$ & $\ldots$ & 4.37 & $4.84 \pm 1.16$ \\
\hline $\mathrm{H} \mathrm{I}$ & 4101 & $2 \mathrm{p}^{2} \mathrm{P}^{\mathrm{o}}-6 \mathrm{~d}^{2} \mathrm{D}$ & 27.8 & $35.0 \pm 4.1$ & 30.4 & $38.7 \pm 4.3$ & 25.5 & $28.2 \pm 3.1$ \\
\hline $\mathrm{H} \mathrm{I}$ & $4340^{f}$ & $2 \mathrm{p}^{2} \mathrm{P}^{\mathrm{o}}-5 \mathrm{~d}^{2} \mathrm{D}$ & 37.8 & $44.7 \pm 2.0$ & 38.8 & $45.8 \pm 2.1$ & 44.0 & $47.1 \pm 2.1$ \\
\hline [O III $]$ & 4363 & $2 \mathrm{p}^{2}{ }^{1} \mathrm{D}_{2}-2 \mathrm{p}^{2}{ }^{1} \mathrm{~S}_{0}$ & 8.22 & $9.6 \pm 1.2$ & 4.84 & $5.68 \pm 0.70$ & 6.79 & $7.25 \pm 0.80$ \\
\hline He I & 4471 & $2 \mathrm{p}^{3} \mathrm{P}^{\mathrm{o}}-4 \mathrm{~d}^{3} \mathrm{D}$ & 7.82 & $8.84 \pm 1.40$ & 4.50 & $5.10 \pm 0.80$ & 6.54 & $6.87 \pm 0.76$ \\
\hline $\mathrm{N}$ III & 4641 & $3 \mathrm{p}^{2} \mathrm{P}_{3 / 2}^{\mathrm{o}}-3 \mathrm{~d}^{2} \mathrm{D}_{5 / 2}$ & $\ldots$ & $\ldots$ & $\ldots$ & $\ldots$ & 2.10 & $2.15 \pm 0.43$ \\
\hline He II & 4686 & $3 \mathrm{~d}^{2} \mathrm{D}-4 \mathrm{f}^{2} \mathrm{~F}^{\mathrm{o}}$ & 1.85 & $1.95 \pm 0.30$ & 1.76 & $1.85 \pm:$ & 2.23 & $2.28 \pm 0.34$ \\
\hline$[\mathrm{Ar}$ IV $]$ & $4711^{i}$ & $3 \mathrm{p}^{3}{ }^{4} \mathrm{~S}_{3 / 2}^{\mathrm{o}}-3 \mathrm{p}^{3}{ }^{2} \mathrm{D}_{5 / 2}^{\mathrm{o}}$ & 2.23 & $2.33 \pm 0.28$ & $\ldots$ & $\ldots$ & 1.55 & $1.58 \pm 0.20$ \\
\hline [Ar IV] & 4740 & $3 \mathrm{p}^{3}{ }^{4} \mathrm{~S}_{3 / 2}^{\mathrm{o}}-3 \mathrm{p}^{3}{ }^{2} \mathrm{D}_{3 / 2}^{\mathrm{o}}$ & 3.30 & $3.43 \pm 0.40$ & $\ldots$ & $\ldots$ & 1.67 & $1.70 \pm 0.22$ \\
\hline $\mathrm{H} \mathrm{I}$ & 4861 & $2 \mathrm{p}^{2} \mathrm{P}^{\mathrm{o}}-4 \mathrm{~d}^{2} \mathrm{D}$ & 100 & 100 & 100 & 100 & 100 & 100 \\
\hline He I & 4922 & $2 \mathrm{p}^{1} \mathrm{P}_{1}^{\mathrm{o}}-4 \mathrm{~d}^{1} \mathrm{D}_{2}$ & 3.03 & $3.00 \pm 1.24$ & $\ldots$ & $\ldots$ & 2.13 & $2.12 \pm 0.84$ \\
\hline [O III $]$ & 4959 & $2 \mathrm{p}^{2}{ }^{1} \mathrm{P}_{1}-2 \mathrm{p}^{2}{ }^{1} \mathrm{D}_{2}$ & 422 & $413 \pm 20$ & 376 & $368 \pm 18$ & 342 & $339 \pm 16$ \\
\hline$[\mathrm{O}$ III] & 5007 & $2 \mathrm{p}^{2}{ }^{1} \mathrm{P}_{2}-2 \mathrm{p}^{2}{ }^{1} \mathrm{D}_{2}$ & 1260 & $1216 \pm 37$ & 1106 & $1068 \pm 32$ & 1033 & $1017 \pm 31$ \\
\hline$[\mathrm{N}$ I $]$ & $5198^{j}$ & $2 \mathrm{p}^{3}{ }^{4} \mathrm{~S}_{3 / 2}^{\mathrm{o}}-2 \mathrm{p}^{3}{ }^{2} \mathrm{D}_{3 / 2}^{\mathrm{o}}$ & $\ldots$ & $\ldots$ & $\ldots$ & $\ldots$ & 1.62 & $1.57 \pm 0.55$ \\
\hline$[\mathrm{N}$ II $]$ & 5755 & $2 \mathrm{p}^{21} \mathrm{D}_{2}-2 \mathrm{p}^{2}{ }^{1} \mathrm{~S}_{0}$ & 2.57 & $2.12 \pm 0.64$ & $\ldots$ & $\ldots$ & 1.10 & $1.02 \pm 0.30$ \\
\hline He I & 5876 & $2 \mathrm{p}^{3} \mathrm{P}^{\mathrm{o}}-3 \mathrm{~d}^{3} \mathrm{D}$ & 21.2 & $17.2 \pm 1.60$ & 16.4 & $13.3 \pm 1.5$ & 17.9 & $16.4 \pm 0.8$ \\
\hline$[\mathrm{O}$ I $]$ & 6300 & $2 \mathrm{p}^{43} \mathrm{P}_{2}-2 \mathrm{p}^{4}{ }^{1} \mathrm{D}_{2}$ & 6.87 & $5.24 \pm 1.30$ & $\ldots$ & $\ldots$ & 7.88 & $7.04 \pm 1.76$ \\
\hline [S III] & $6312^{k}$ & $3 \mathrm{p}^{2}{ }^{1} \mathrm{D}_{2}-3 \mathrm{p}^{2}{ }^{1} \mathrm{~S}_{0}$ & $\ldots$ & $\ldots$ & $\ldots$ & $\ldots$ & 2.03 & $1.81 \pm 0.56$ \\
\hline$[\mathrm{O}$ I $]$ & 6363 & $2 \mathrm{p}^{43} \mathrm{P}_{1}-2 \mathrm{p}^{4}{ }^{1} \mathrm{D}_{2}$ & 3.73 & $2.82 \pm 1.26$ & $\ldots$ & $\cdots$ & 2.33 & $2.08 \pm 0.80$ \\
\hline$[\mathrm{N}$ II $]$ & 6548 & $2 \mathrm{p}^{2}{ }^{3} \mathrm{P}_{1}-2 \mathrm{p}^{2}{ }^{1} \mathrm{D}_{2}$ & 33.1 & $24.4 \pm 2.9$ & 5.91 & $4.36 \pm 0.52$ & 28.1 & $24.8 \pm 3.0$ \\
\hline $\mathrm{H} \mathrm{I}$ & 6563 & $2 \mathrm{p}^{2} \mathrm{P}^{\mathrm{o}}-2 \mathrm{~d}^{2} \mathrm{D}$ & 357 & $263 \pm 1$ & 338 & $249 \pm 1$ & 341 & $300 \pm 1$ \\
\hline$[\mathrm{N}$ II $]$ & 6583 & $2 \mathrm{p}^{2}{ }^{3} \mathrm{P}_{2}-2 \mathrm{p}^{2}{ }^{1} \mathrm{D}_{2}$ & 96.5 & $71.0 \pm 7.5$ & 18.0 & $13.2 \pm 1.5$ & 87.3 & $76.8 \pm 8.4$ \\
\hline He I & $6678^{l}$ & $2 \mathrm{p}^{1} \mathrm{P}_{1}^{\mathrm{o}}-3 \mathrm{~d}^{1} \mathrm{D}_{2}$ & 5.39 & $3.90 \pm 0.58$ & $\ldots$ & $\ldots$ & 5.11 & $4.47 \pm 0.67$ \\
\hline [S II] & 6716 & $3 \mathrm{p}^{3}{ }^{4} \mathrm{~S}_{3 / 2}^{\mathrm{o}}-3 \mathrm{p}^{3}{ }^{2} \mathrm{D}_{5 / 2}^{\mathrm{o}}$ & 10.3 & $7.45 \pm 0.83$ & $\ldots$ & $\ldots$ & 10.1 & $8.8 \pm 1.0$ \\
\hline$[\mathrm{S}$ II $]$ & 6731 & $3 \mathrm{p}^{3}{ }^{4} \mathrm{~S}_{3 / 2}^{\mathrm{o}}-3 \mathrm{p}^{32} \mathrm{D}_{3 / 2}^{\mathrm{o}}$ & 13.6 & $9.8 \pm 1.0$ & $\ldots$ & $\ldots$ & 15.4 & $13.5 \pm 1.0$ \\
\hline$[\mathrm{Ar} \mathrm{V}]$ & 7005 & $3 \mathrm{p}^{23} \mathrm{P}_{2}-3 \mathrm{p}^{2}{ }^{1} \mathrm{D}_{2}$ & $\ldots$ & $\ldots$ & $\ldots$ & $\ldots$ & 0.50 & $0.42 \pm 0.11$ \\
\hline He I & 7065 & $2 \mathrm{p}^{3} \mathrm{P}^{\mathrm{o}}-3 \mathrm{~s}^{3} \mathrm{~S}$ & 9.90 & $6.80 \pm 0.86$ & $\ldots$ & $\ldots$ & 11.6 & $10.0 \pm 1.3$ \\
\hline [Ar III] & 7136 & $3 p^{43} \mathrm{P}_{2}-3 \mathrm{p}^{4}{ }^{1} \mathrm{D}_{2}$ & 34.5 & $23.4 \pm 3.0$ & $\ldots$ & $\ldots$ & 20.9 & $17.8 \pm 2.13$ \\
\hline$\left[\begin{array}{ll}\mathrm{O} & \mathrm{II}\end{array}\right]$ & 7320 & $2 \mathrm{p}^{32} \mathrm{D}_{5 / 2}^{\mathrm{o}}-2 \mathrm{p}^{3}{ }^{2} \mathrm{P}_{3 / 2}^{\mathrm{o}}$ & $\ldots$ & $\ldots$ & $\ldots$ & $\ldots$ & 5.77 & $4.87 \pm 0.73$ \\
\hline$[\mathrm{O} \quad \mathrm{II}]$ & $7330^{m}$ & $2 \mathrm{p}^{32} \mathrm{D}_{3 / 2}^{\mathrm{o}}-2 \mathrm{p}^{32} \mathrm{P}_{3 / 2}^{\mathrm{o}}$ & $\ldots$ & $\ldots$ & $\ldots$ & $\ldots$ & 5.94 & $5.01 \pm 0.75$ \\
\hline [Ar III] & 7751 & $3 \mathrm{p}^{43} \mathrm{P}_{1}-3 \mathrm{p}^{41} \mathrm{D}_{2}$ & 11.1 & $6.93 \pm 2.43$ & $\ldots$ & $\ldots$ & 4.29 & $3.53 \pm 1.24$ \\
\hline \multicolumn{3}{|l|}{$c(\mathrm{H} \beta)$} & \multirow{2}{*}{\multicolumn{2}{|c|}{$\begin{array}{c}0.451 \\
-14.94\end{array}$}} & \multirow{2}{*}{\multicolumn{2}{|c|}{$\begin{array}{c}0.450 \\
-15.13\end{array}$}} & \multirow{2}{*}{\multicolumn{2}{|c|}{$\begin{array}{c}0.186 \\
-15.10\end{array}$}} \\
\hline $\log F(\mathrm{H}$ & & & & & & & & \\
\hline
\end{tabular}

${ }^{a}$ Fluxes (also the intensities) are normalized such that $\mathrm{H} \beta=100$. Emission lines that were not detected in the spectra of PN5, PN6 or PN7 are not presented in the table for the three targets.

${ }^{b}$ A blend of the $\mathrm{O}$ II $\lambda 3726\left(2 \mathrm{p}^{3}{ }^{4} \mathrm{~S}_{3 / 2}^{\mathrm{o}}-2 \mathrm{p}^{3}{ }^{2} \mathrm{D}_{3 / 2}^{\mathrm{o}}\right)$ and $\lambda 3729\left(2 \mathrm{p}^{3}{ }^{4} \mathrm{~S}_{3 / 2}^{\mathrm{o}}-2 \mathrm{p}^{3}{ }^{2} \mathrm{D}_{5 / 2}^{\mathrm{o}}\right)$ lines.

${ }^{c}$ Blended with the He I $\lambda 3888\left(2 \mathrm{~s}^{3} \mathrm{~S}-3 \mathrm{p}^{3} \mathrm{P}^{\mathrm{o}}\right)$ line.

${ }^{d}$ Blended with the H I $\lambda 3970\left(2 \mathrm{p}^{2} \mathrm{P}^{\mathrm{o}}-7 \mathrm{~d}^{2} \mathrm{D}\right)$ and He I $\lambda 3965\left(2 \mathrm{~s}^{1} \mathrm{~S}_{0}-4 \mathrm{p}^{1} \mathrm{P}_{1}^{\mathrm{o}}\right)$ lines.

e Blended with the $\mathrm{O}$ II M10 3p ${ }^{4} \mathrm{D}^{\mathrm{o}}-3 \mathrm{~d}^{4} \mathrm{~F}$ and $\mathrm{C}$ III M16 $4 \mathrm{f}^{3} \mathrm{~F}^{\mathrm{o}}-5 \mathrm{~g}{ }^{3} \mathrm{G}$, and $[\mathrm{S} \mathrm{II}] 4076\left(3 \mathrm{p}^{3}{ }^{4} \mathrm{~S}_{3 / 2}^{\mathrm{o}}-3 \mathrm{p}^{3}{ }^{2} \mathrm{P}_{1 / 2}^{\mathrm{o}}\right)$ lines.

$f$ Corrected for the flux contribution from the blended He II $\lambda 4338\left(4 \mathrm{f}^{2} \mathrm{~F}^{\mathrm{o}}-10 \mathrm{~g}^{2} \mathrm{G}\right)$ line. Fluxes of $\mathrm{H} \beta$ and $\mathrm{H} \alpha$ have also been corrected for the blended He II $\lambda 4859\left(4 \mathrm{f}^{2} \mathrm{~F}^{\mathrm{o}}-8 \mathrm{~g}^{2} \mathrm{G}\right)$ and $\lambda 6560\left(4 \mathrm{f}^{2} \mathrm{~F}^{\mathrm{o}}-6 \mathrm{~g}^{2} \mathrm{G}\right)$ lines, respectively.

$g$ Blended with the $\mathrm{N}$ III $\lambda 4634$ and $\lambda 4642$ lines of multiplet M2 3p $2 \mathrm{P}^{\mathrm{o}}-3 \mathrm{~d}^{2} \mathrm{D}$ and the $\mathrm{O}$ II $\lambda 4642$ and $\lambda 4639$ lines of multiplet M1 $3 \mathrm{~s}^{4} \mathrm{P}$ $-3 \mathrm{p}^{4} \mathrm{D}^{\mathrm{o}}$.

$h$ Probably blended with the C IV $\lambda 4658\left(\mathrm{M} 85 \mathrm{~g}^{2} \mathrm{G}-6 \mathrm{~h}^{2} \mathrm{H}^{\circ}\right)$ line for the case of PN2.

${ }^{i}$ Corrected for the flux contribution from the blended He I $\lambda 4713\left(2 \mathrm{p}^{3} \mathrm{P}^{\mathrm{o}}-4 \mathrm{~s}^{3} \mathrm{~S}\right)$ line.

$j$ Blended with [N I] $\lambda 5200\left(2 \mathrm{p}^{3}{ }^{4} \mathrm{~S}_{3 / 2}^{\mathrm{o}}-2 \mathrm{p}^{3}{ }^{2} \mathrm{D}_{5 / 2}^{\mathrm{o}}\right.$.

$k$ Corrected for the flux contribution from the blended He II $\lambda 6311\left(5 \mathrm{~g}{ }^{2} \mathrm{G}-16 \mathrm{~h}^{2} \mathrm{H}^{\mathrm{o}}\right)$ line.

${ }^{l}$ Corrected for the flux contribution from the blended He II $\lambda 6683\left(5 \mathrm{~g}^{2} \mathrm{G}-13 \mathrm{~h}{ }^{2} \mathrm{H}^{\circ}\right)$ line.

$m$ Blended with the [Ar IV] $\lambda 7331\left(3 \mathrm{p}^{3}{ }^{2} \mathrm{D}_{5 / 2}^{\mathrm{o}}-{ }^{2} \mathrm{P}_{1 / 2}^{\mathrm{o}}\right)$ line, whose flux contribution is negligible $(<1 \%)$.

${ }^{n}$ In unit of erg $\mathrm{cm}^{-2} \mathrm{~s}^{-1}$ in the extracted spectra. 
TABLE 3

References for Atomic Data

\begin{tabular}{|c|c|c|}
\hline \multirow[t]{2}{*}{ Ion } & \multicolumn{2}{|c|}{ CELs } \\
\hline & Transition probabilities & Collision strengths \\
\hline $\mathrm{N}^{+}$ & Bell et al. (1995) & Stafford et al. (1994) \\
\hline $\mathrm{O}^{+}$ & Zeippen (1987) & Pradhan et al. $(2006)$ \\
\hline $\mathrm{O}^{2+}$ & Storey \& Zeippen (2000) & Lennon \& Burke (1994) \\
\hline $\mathrm{Ne}^{2+}$ & Landi \& Bhatia (2005) & McLaughlin \& Bell $(2000)$ \\
\hline $\mathrm{S}^{+}$ & Keenan et al. $(1993)$ & Ramsbottom et al. $(\overline{1996})$ \\
\hline $\mathrm{S}^{2+}$ & Mendoza \& Zeippen (1982a) & Tayal \& Gupta $(1999)$ \\
\hline $\mathrm{Ar}^{2+}$ & Biémont \& Hansen (1986) & Galavis et al. (1995) \\
\hline $\mathrm{Ar}^{3+}$ & Mendoza \& Zeippen (1982b) & Ramsbottom et al. (1997) \\
\hline $\mathrm{Ar}^{4+}$ & Mendoza \& Zeippen (1982a) & Mendoza $(1983)$ \\
\hline \multirow[t]{2}{*}{ Ion } & \multicolumn{2}{|c|}{ ORLs } \\
\hline & Effective recombination coeff & Comments \\
\hline H I & Storey \& Hummer 1995$)$ & Case B \\
\hline He I & Porter et al. $(2012)$ & Case B \\
\hline He II & Storey \& Hummer (1995) & Case B \\
\hline $\mathrm{C}_{\mathrm{II}}$ & Davey et al. $(2000)$ & Case B \\
\hline
\end{tabular}

were used for density diagnostics. The [O II $] \lambda 3727$ line is a blend of the $\lambda \lambda 3726,3729$ doublet. Since the [O II] $\lambda \lambda 7320,7330$ auroral lines were detected in the spectra of PN1-4 and PN7, we also used the [O II] $\lambda 3727 /(\lambda 7320$ $+\lambda 7330)$ line ratio to estimate electron temperatures for these five objects. The weak [S II] $\lambda 4068$ line is blended with the even weaker $[\mathrm{S} \mathrm{II}] \lambda 4076$ line as well as with the O II M10 $3 \mathrm{p}^{4} \mathrm{D}^{\mathrm{o}}-3 \mathrm{~d}^{4} \mathrm{~F}$ and C III M16 $4 \mathrm{f}^{3} \mathrm{~F}^{\mathrm{o}}-5 \mathrm{~g}^{3} \mathrm{G}$ lines. The electron temperatures derived from the $[\mathrm{S} \mathrm{II}]$ $(\lambda 6716+\lambda 6731) / \lambda 4072$ ratio are highly uncertain.

Figure 6 shows the plasma diagnostic diagrams for six of our PNe. The diagram of PN6 is not presented because only the $[\mathrm{O}$ III $] \lambda 4363$ diagnostic line was detected in the spectrum of this object. These diagnostic diagrams are based on CELs and were created by solving the level population equations for five-level atomic models using EQUIB, a FORTRAN code originally developed by Howarth \& Adams (1981) to solve the equations of statistical equilibrium in multi-level atoms and yield level populations and line emissivities for a specified physical condition, appropriate to the zones where the ions are expected to exist. The atomic data used by the EQUIB code were updated manually. References for the atomic data set used for plasma diagnostics based on CELs, as well as for ionic abundance determinations (Section 3.4.1), are presented in Table 3. Results of our plasma diagnostics are presented in Table 4

The uncertainties in the electron temperatures and densities (Table 4) were estimated through the propagation of uncertainties in the emission line intensities, and do not include the contribution from systematic effects. As discussed in Section 3.1, the error contributions from the systematic effects, such as reddening, flux calibration, and line blending, are expected to be small. The atomic data used for plasma diagnostics are mostly updated (Table 3). It is difficult to quantitatively estimate the systematic uncertainties introduced by the atomic data. We expect that the atomic data adopted are reliable, since these calculations were improved over the previous work (see the discussion in Section 7 of Kwitter et al. 2014 contributed by X.-W. Liu and X. Fang).

Figure 6 shows that the [O III] line ratio is an excellent temperature diagnostic, and variation of the electron density does not change the resultant electron temperature much. Uncertainties in the electron temperatures derived from the $[\mathrm{N} \mathrm{II}]$ line ratio are much larger than those of the $[\mathrm{O}$ III $]$ temperatures, mainly due to weakness of the [N II] $\lambda 5755$ auroral line. Besides, the [N II] nebular-to-auroral line ratio has also slight density dependence (Figure 6). The [O II] nebular-to-auroral line ratio is sensitive to both temperature and density, and thus not an ideal temperature indicator. For some targets, the $[\mathrm{N} \mathrm{II}]$ temperature is higher than the [O $\mathrm{O}$ III] temperature, which is likely due to the overestimated intensity of the very weak $\lambda 5755$ line. Only in one object (PN7) the $[\mathrm{N} \mathrm{II}]$ temperature seems to be meaningfully different from the $[\mathrm{O}$ III] temperature (Table 4). The [O III] temperature is thus adopted in the abundance calculations for all PNe. We are aware that in a PN there are different ionization zones, as represented by the $\mathrm{O}^{2+}$ and $\mathrm{N}^{+}$ions, and using only the $[\mathrm{O}$ III] temperature may introduce extra errors in the ionic abundances for the singly ionized species. In this respect, the actual uncertainties in the $\mathrm{N}^{+} / \mathrm{H}^{+}, \mathrm{O}^{+} / \mathrm{H}^{+}$and $\mathrm{S}^{+} / \mathrm{H}^{+}$abundance ratios might be slightly larger than those given in Table 5 . Recently, in a spectroscopic study of Galactic PNe, $\mathrm{Du}-$ four et al. (2015) adopted a constant [N II] temperature, which was carefully derived by Kaler (1986) for the case where there is no detection of the $[\mathrm{N}$ II $] \lambda 5755$ line but He II $\lambda 4686$ is present.

In a few cases of our PN sample, there is large difference between the electron density derived from the [S II] line ratio and that derived from [Ar IV], as shown in Table 4. It might be that in our PNe there are lowionization regions where the [S II] density is more appropriate, whereas the [Ar IV] density applies in the higher ionization regions. Thus we adopted the [S II] densities for the ionic-abundance calculations of the low ionization species and adopted the [Ar IV] densities for the high ionization species (Section 3.4.1). Measurements of the relatively faint [Ar IV] lines in PN5 are of large uncertainty due to the noise, and for this object we adopted the $[\mathrm{S}$ II] density.

An electron density of $\sim 2000 \mathrm{~cm}^{-3}$, typical for most of our targets, was assumed for PN6 because neither the [S II] nor the [Ar IV] doublet was detected in this PN. In order to check how much the assumed electron density would affect the resultant ionic abundances, we calculated ionic abundances for two density cases, $10^{3}$ and $10^{4} \mathrm{~cm}^{-3}$, at $9500 \mathrm{~K}$, the [O $\left.\mathrm{III}\right]$ temperature derived for PN6. The $\mathrm{N}^{+} / \mathrm{H}^{+}$ionic abundance ratio derived at the two density cases differ by $\sim 10 \%$, while both the $\mathrm{O}^{2+} / \mathrm{H}^{+}$ and the $\mathrm{Ne}^{2+} / \mathrm{H}^{+}$ratio differ by $<4 \%$; the $\mathrm{O}^{+} / \mathrm{H}^{+}$abundance ratio derived from the $[\mathrm{O}$ II $] \lambda 3727$ line differ by nearly $50 \%$ at the two densities. These differences in the CEL ionic abundances at the two density cases are due to the large differences in critical densities, as discussed in Paper I. For the helium recombination lines, differences in ionic abundances derived at the two density cases are negligible $(<6 \%$; Porter et al. 2012$)$.

The relatively high electron temperature and density of PN3 (Table 4 ) indicate that this target may be young (compact) compared to other PNe. The [O III] $\lambda 4363$ auroral line in PN3 is stronger than other PNe. PN3 also has strong He II $\lambda 4686$ line $(\sim 0.2 \mathrm{H} \beta)$, while this line is much fainter in the spectra of the other targets (Table 2). The above information points to the high-excitation na- 


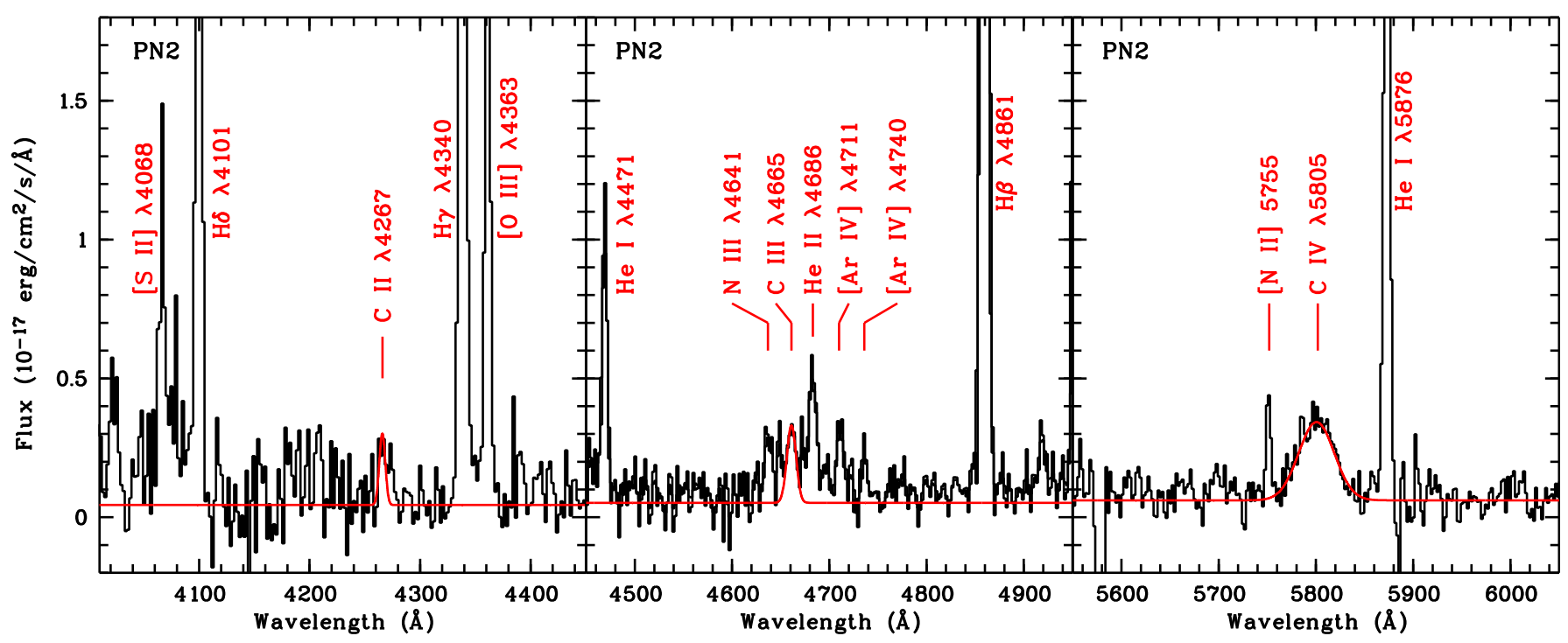

FIG. 5.- The carbon lines detected in the spectrum of PN2: C II $\lambda 4267$ (left), C III $\lambda 4665$ (middle), and C IV $\lambda 5805$ (right). The broad C IV $\lambda 5805$ feature is emitted by a Wolf-Rayet type central star. The red continuous curves are Gaussian profile fits to the carbon lines, with FWHM=43 $\AA$ for the $\lambda 5805$ line. An FWHM of $6 \AA$ is assumed for the $\lambda 4267$ line and $\sim 11 \AA$ for $\lambda 4665$.

ture of PN3. This high excitation could due to either a very hot central star or the low metallicity, or both. The relatively high density in PN3 may also cause collisional de-excitation of the heavy element ions, which makes the radiation cooling inefficient and as a consequence, produces relatively higher electron temperature.

The intensity ratio of two He I optical recombination lines that originate from two atomic levels with different excitation energies is sensitive to the electron temperature (although not so acutely sensitive to $T_{\mathrm{e}}$ as the CELs), and thus can also be used as a temperature diagnostic. The temperature diagnostics for gaseous nebulae based on the He I recombination lines were constructed by Zhang et al. (2005) using the theoretical He I line emissivities calculated by Benjamin et al. (1999). We have determined the He I line temperatures for our PNe following the diagnostic method of Zhang et al. (2005) and the results are presented in Table 4. Generally the electron temperatures derived from the He I line ratios, designated $T_{\mathrm{e}}(\mathrm{He} \mathrm{I})$, are lower than those derived from the heavy element CELs, $T_{\mathrm{e}}(\mathrm{CEL})$, except PN4 and PN7, whose He I $\lambda 6678 / \lambda 4471$ ratio yields higher temperatures. This is in line with the temperature sequence, $T_{\mathrm{e}}(\mathrm{CEL}) \gtrsim T_{\mathrm{e}}(\mathrm{H} \mathrm{I}) \gtrsim T_{\mathrm{e}}$ (He I), so far observed in more than 100 Galactic PNe through deep spectroscopy (e.g., McNabb et al. 2013, see also recent reviews by Liu 2006 , 2012). Zhang et al. (2005) studied 48 Galactic PNe and found that the $T_{\mathrm{e}}(\mathrm{He} \mathrm{I})$ values are significantly lower than the electron temperatures derived from the $\mathrm{H}$ i Balmer jump, $T_{\mathrm{e}}(\mathrm{H} \mathrm{I})$, with an average difference of $4000 \mathrm{~K}$. The significant difference between the electron temperatures of PNe derived from the CELs and the $\mathrm{H}$ I (also He I) recombination spectrum is the renowned "temperature discrepancy" in nebular astrophysics (e.g., Peimbert 1971. Liu \& Danziger 1993). Investigation of this subject has never been extended to extragalactic PNe due to limited S/N. The spectral quality of our PN targets also prevented further study of the problem.

\subsection{Abundances}

\subsubsection{Ionic Abundances}

In order to derive ionic abundances of the heavy elements from CELs, the equations of statistical equilibrium were solved using the program EQUIB to determine the population of the upper level of a given transition. References of the atomic data used for ionic abundance calculations are summarized in Table 3 . The electron temperatures and densities (Table 4) obtained from plasma diagnostics (Section 3.3) were assumed in the abundance calculations. Ionic abundances derived using the extinction-corrected fluxes of the lines detected in the spectra of the seven PNe are presented in Table 5 .

Helium - The $\mathrm{He}^{+} / \mathrm{H}^{+}$ionic abundances were derived from the He I $\lambda \lambda 3888,4471,5876$ and 6678 lines (Table 5), which are among the strongest helium recombination lines in the optical. The effective recombination coefficients of the He I lines calculated by Porter et al. (2012) were adopted in the abundance determination. The He I $\lambda 3888$ line is blended with H I $\lambda 3889(n=$ $2-8$ ), which contributes $\sim 50-60 \%$ of the total flux. We corrected for the blended $\mathrm{H}$ I line flux using the theoretical H I $\lambda 3889 / \lambda 4861$ line ratio at $10000 \mathrm{~K}$ and $10^{4} \mathrm{~cm}^{-3}$ in Case B (Storey \& Hummer 1995). However, the ionic abundance yielded by the corrected $\lambda 3888$ line flux is lower than those derived from the $\lambda \lambda 4471,5876$ and 6678 lines for our targets (Table 5). Such significant difference between the $\mathrm{He}^{+} / \mathrm{H}^{+}$abundance derived from the $\lambda 3888$ line and those derived from the other three He I lines is difficult to explain. One possibility is the effect of self-absorption due to large population in the $1 \mathrm{~s} 2 \mathrm{~s}^{3} \mathrm{~S}_{1}$ metastable level of He I (e.g., Robbins 1968, Benjamin et al. 2002). The limited number of well-detected He I lines in our sample prevents further study of such effect. The only agreement in the $\mathrm{He}^{+} / \mathrm{H}^{+}$abundances in found in PN6. The $\mathrm{He}^{+} / \mathrm{H}^{+}$abundance derived from the He I $\lambda 5876$ line is adopted for our sample, given that this line is stronger than the other He I optical recombination lines and thus best observed.

The $\mathrm{He}^{2+} / \mathrm{H}^{+}$abundance was derived from the He II 
TABLE 4

Plasma Diagnostics

\begin{tabular}{|c|c|c|c|c|c|c|c|}
\hline Diagnostic Ratio & PN1 & $\mathrm{PN} 2$ & PN3 & $\mathrm{PN} 4$ & PN5 & PN6 & PN7 \\
\hline $\begin{array}{l}{[\mathrm{O} \text { iII] }(\lambda 4959+\lambda 5007) / \lambda 4363} \\
{\left[\begin{array}{ll}\mathrm{N} & \mathrm{II}\end{array}(\lambda 6548+\lambda 6583) / \lambda 5755\right.} \\
{[\mathrm{O} \text { II] } \lambda 3727 /(\lambda 7320+\lambda 7330)} \\
\text { [S II] }(\lambda 6716+\lambda 6731) / \lambda 4072^{a} \\
\text { He I } \lambda 5876 / \lambda 4471 \\
\text { He I } \lambda 6678 / \lambda 4471 \\
\text { Adopted }^{b}\end{array}$ & $\begin{array}{c}11000 \pm 400 \\
14300 \pm 2400 \\
>20000 \\
\ldots \\
5300 \pm 2000 \\
5200 \pm 2500 \\
11000 \pm 400\end{array}$ & $\begin{array}{c}11600 \pm 500 \\
11300 \pm 1500 \\
>20000 \\
\ldots \\
6100 \pm 2000 \\
3400 \pm 3000 \\
11600 \pm 500\end{array}$ & $\begin{array}{c}12900 \pm 550 \\
12300 \pm 1300 \\
15200 \pm 2200 \\
12000 \pm 6700 \\
8000 \pm 2000 \\
9700 \pm 3000 \\
12900 \pm 550\end{array}$ & $\begin{array}{c}T_{\mathrm{e}}(\mathrm{K}) \\
11600 \pm 700 \\
12400 \pm 2000 \\
14600 \pm 3000 \\
15400 \pm 8000 \\
<5000 \\
12500 \pm 7500 \\
11600 \pm 500\end{array}$ & $\begin{array}{c}10600 \pm 700 \\
11800 \pm 2000 \\
\cdots \\
\cdots \\
\cdots \\
\cdots \\
10600 \pm 400\end{array}$ & $\begin{array}{c}9500 \pm 600 \\
\ldots \\
\ldots \\
\cdots \\
\cdots \\
\cdots \\
9500 \pm 400\end{array}$ & $\begin{array}{c}10300 \pm 350 \\
9100 \pm 1000 \\
11000 \pm 1100 \\
10000 \pm 8600 \\
<5000 \\
15000 \pm 6000 \\
10300 \pm 350\end{array}$ \\
\hline $\begin{array}{l}{[\mathrm{S} \text { II] } \lambda 6716 / \lambda 6731} \\
{[\mathrm{Ar} \text { IV] } \lambda 4711 / \lambda 4740}\end{array}$ & $\begin{array}{c}7000 \pm 2300 \\
3000 \pm 200\end{array}$ & $\begin{array}{c}5900 \pm 2100 \\
1900 \pm 300\end{array}$ & $\begin{array}{c}10200 \pm 4400 \\
7700 \pm 200\end{array}$ & $\begin{array}{c}N_{\mathrm{e}}\left(\mathrm{cm}^{-3}\right) \\
2000 \pm 1000 \\
2900 \pm 400\end{array}$ & $\begin{array}{c}1700 \pm 800 \\
9400 \pm 2000\end{array}$ & $\begin{array}{c}2000^{c} \\
\ldots\end{array}$ & $\begin{array}{c}2900 \pm 1200 \\
5400 \pm 600\end{array}$ \\
\hline
\end{tabular}

${ }^{a}$ A blend of [S II] $\lambda \lambda 4068,4076$ and the O II M10 3p ${ }^{4} \mathrm{D}^{\circ}-3 \mathrm{~d}^{4} \mathrm{~F}$ and C III M16 $4 \mathrm{f}^{3} \mathrm{~F}^{\mathrm{o}}-5 \mathrm{~g}{ }^{3} \mathrm{G}$ lines.

$b$ The electron temperatures derived from the [O III] line ratio are adopted.

${ }^{c}$ An assumed electron density for PN6 because neither [S II] nor [Ar IV] lines were observed.
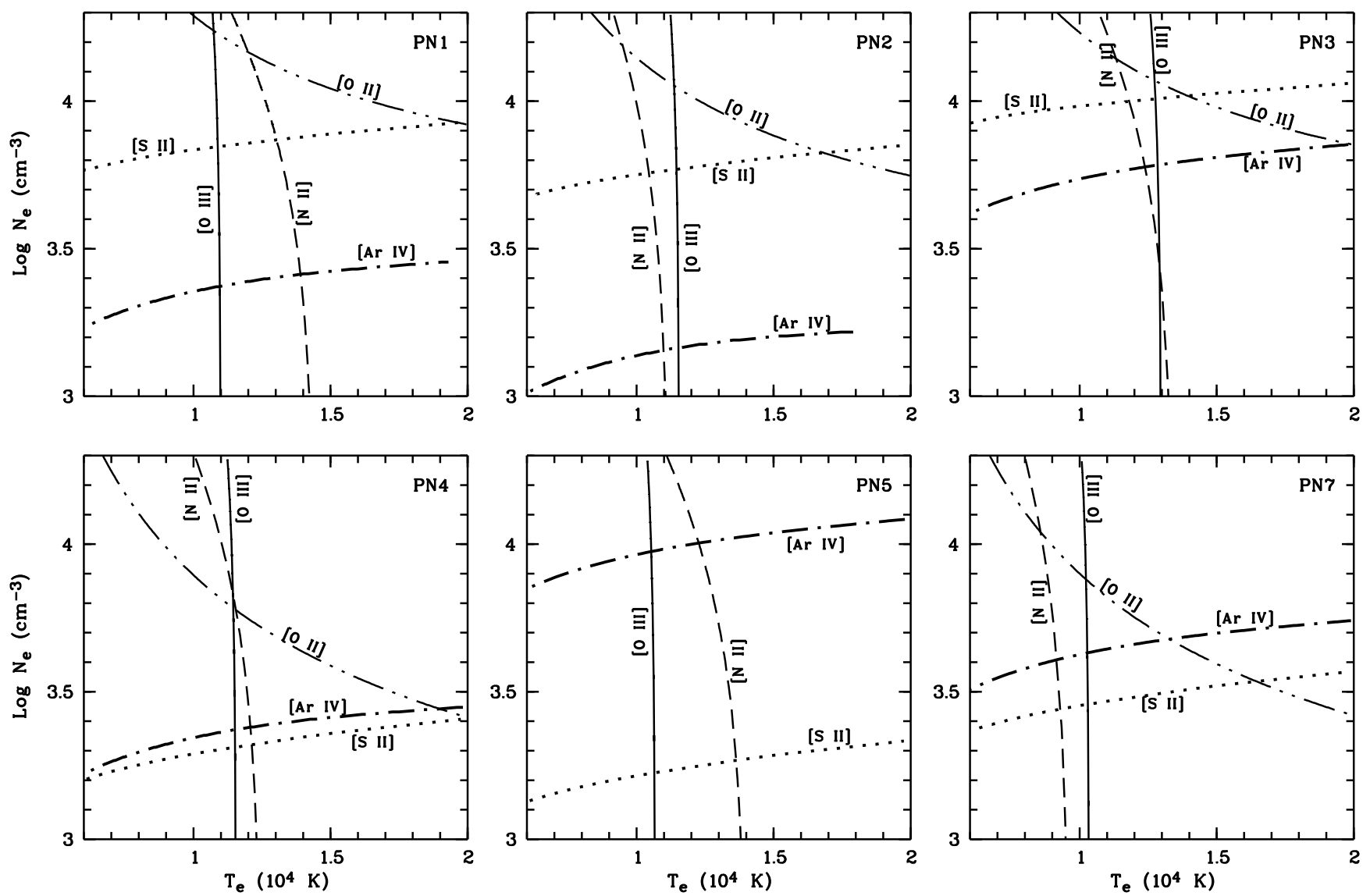

FIG. 6.- Plasma diagnostic diagrams for PN1 (top left), PN2 (top middle), PN3 (top right), PN4 (bottom left), PN5 (bottom middle) and PN7 (bottom right). Different line types represent the temperature or density diagnostics using different ions (see Table 4 for line ratios). The dotted line is the $[\mathrm{S} \mathrm{II}] \lambda 6716 / \lambda 6731$ density-diagnostic curve. The diagram of PN6 is not presented due to the lack of diagnostics (see text for details). 
$\lambda 4686$ line, which was detected in the spectra of all targets except PN6, for which an upper limit of the line flux was estimated from the standard deviation of the local continuum. This upper limit value is close to the He II line fluxes of other faint PNe. Another He II line $\lambda 5411$ was only detected in PN3, which also has the strongest $\lambda 4686$ line. The $\mathrm{He}^{2+} / \mathrm{H}^{+}$abundance of PN3 is higher than those of the other PNe by nearly one order of magnitude, indicating that this $\mathrm{PN}$ is highly excited. The effective recombination coefficients of the He II line used in the abundance determination was adopted from the hydrogenic calculations of Storey \& Hummer (1995).

Oxygen - The $\mathrm{O}^{2+} / \mathrm{H}^{+}$abundances derived from the [O III] nebular lines were adopted for our PNe. The $\mathrm{O}^{2+} / \mathrm{H}^{+}$abundance of PN3 is lower than those of the other PNe. Given that $\mathrm{O}^{2+}$ is one of the dominant coolants in $\mathrm{PNe}$, the low $\mathrm{O}^{2+} / \mathrm{H}^{+}$ionic abundance in PN3 makes the cooling inefficient and as a consequence, the highest electron temperature of this PN (Table 4 ) and thus the strongest He II $\lambda 4686$ line. The [O II] $\lambda 3727$ (a blend of the $\lambda \lambda 3726,3729$ doublet) line is much stronger than the [O II] $\lambda \lambda 7320,7330$ auroral lines and thus better detected. The $\mathrm{O}^{+} / \mathrm{H}^{+}$abundance derived from the $\lambda 3727$ line is adopted for all targets.

Nitrogen, Neon - The weak [N II] $\lambda 5755$ auroral line was detected in most of the PNe. The $\mathrm{N}^{+} / \mathrm{H}^{+}$abundance derived from the $\lambda 6583$ line was adopted for our targets. The [Ne III] $\lambda 3967$ line is blended with $\mathrm{H}$ I $\lambda 3970$, and thus yields higher $\mathrm{Ne}^{2+} / \mathrm{H}^{+}$abundance than the $\lambda 3868$ line, which is free of line blending. The abundance derived from [Ne III] $\lambda 3868$ is adopted for all targets.

Argon, Sulfur - The [Ar IV] $\lambda \lambda 4711,4740$ doublet is blended with the [Ne IV] $\lambda \lambda 4714,15$ and 4724,25 lines of the $2 \mathrm{p}^{3}{ }^{2} \mathrm{D}^{\mathrm{o}}-{ }^{2} \mathrm{P}^{\mathrm{o}}$ transition. The flux contribution of these [Ne IV] lines are negligible (1-2\%). However, the blended He I $\lambda 4713\left(2 \mathrm{p}^{3} \mathrm{P}^{\mathrm{o}}-4 \mathrm{~s}^{3} \mathrm{~S}\right)$ contributes $30-40 \%$ to the total flux at $\lambda 4711$ (Section 3.1). The corrected flux of the [Ar IV] $\lambda 4711$ line yielded an $\mathrm{Ar}^{3+} / \mathrm{H}^{+}$consistent with that derived from the $\lambda 4740$ line. The [Ar III] $\lambda \lambda 7136,7751$ nebular lines were detected in five PNe. The $\lambda 7751$ line is located at the red end of the spectra and its flux could be unreliable due to low efficiency of the instrument. Thus the $\mathrm{Ar}^{2+} / \mathrm{H}^{+}$abundance derived from the stronger $\lambda 7136$ line is adopted for our targets. The $[$ Ar V] $\lambda 7005$ line was detected in PN2, PN3 and $\mathrm{PN} 7$, and the $\mathrm{Ar}^{4+} / \mathrm{H}^{+}$abundance was derived using this line (Table 5). The $\mathrm{S}^{+} / \mathrm{H}^{+}$abundance derived from the [S II] $\lambda 6731$ line is adopted for our PNe, given that $\lambda 6731$ is the stronger line of the doublet. The [S III] $\lambda 6312$ line is detected in four of our targets and the $\mathrm{S}^{2+} / \mathrm{H}^{+}$abundance was derived using this line.

Carbon - The C II $\lambda 4267$ optical recombination line was detected in the spectrum of PN2 (Figure 5). Accurate measurements of the optical recombination lines of heavy elements were difficult due to weakness $\left(\leq 10^{-4}-10^{-3}\right.$ of the $\mathrm{H} \beta$ flux). In the past two decades, deep spectroscopy with an aid of modern high-efficiency and large-format linear detectors has enabled detection of many optical recombination lines of heavy element ions (mainly $\mathrm{C}$ II,
N II, O II, Ne II) in the spectra of Galactic PNe, and new astrophysics have been revealed through studies of these weak lines (e.g., Liu et al. 2000, Fang \& Liu 2013; see also the reviews Liu 2006, 2012). The weak optical recombination lines of heavy elements have previously been detected in the spectra of extragalactic PNe, e.g., in one M31 PNe by Balick et al. (2013, Table 2) and in the Magellanic Cloud PNe by Leisy \& Dennefeld (2006) and Shaw et al. (2010). The C II $\lambda 4267$ line is produced by radiative recombination only, and thus is used to derive the $\mathrm{C}^{2+} / \mathrm{H}^{+}$abundance in this paper. The $\mathrm{C}^{2+} / \mathrm{H}^{+}$abundance ratio of target $\mathrm{PN} 2$ is $1.7 \pm 0.8 \times 10^{-3}$ (Table 5), which agrees with the target observed by Balick et al. (2013) within errors.

The [O III] electron temperature of PN2 (11600 K) was assumed when calculating the recombination line $\mathrm{C}^{2+} / \mathrm{H}^{+}$abundance. This is the same strategy used by Balick et al. (2013). However, deep spectroscopic observations of numerous Galactic PNe have provided evidence that the optical recombination lines of heavy elements come from plasma as cold as $<1000 \mathrm{~K}$ (e.g., Liu 2006, Liu et al. 2006, Fang \& Liu 2013, McNabb et al. 2013). If this is also true for the M31 PNe, then our $\mathrm{C}^{2+} / \mathrm{H}^{+}$abundance ratio could be overestimated. At an electron temperature of $1000 \mathrm{~K}$, the calculated $\mathrm{C}^{2+} / \mathrm{H}^{+}$abundance ratio for $\mathrm{PN} 2$ is $1.8 \times 10^{-4}$, lower than the value calculated using the [O III] temperature by nearly one order of magnitude. The C II effective recombination coefficients of Davey et al. (2000) was adopted for the abundance calculations.

The abundance errors following the ionic abundance ratios in Table 5 were mainly propagated from the measurement errors of the extinction-corrected relative line intensities given in Table 2. Since the [O III] $\lambda 4363 \mathrm{au}-$ roral line was well detected in the spectra of all targets (Figures 4), the errors in the [O III] electron temperatures are generally small (Table 4 ) and not considered in the ionic abundance calculations. However, as discussed in Section 3.3, adopting the [O III] temperature for all ionic species might introduce extra uncertainties in ionic abundance calculations, especially for the low ionization species, as PNe have ionization structures. This uncertainty is difficult to assess due to the limited number of reliable temperature diagnostics. The errors in the electron temperature potentially usually have large impact on in the abundances derived from CELs, given that the emissivity of a CEL has an exponential dependence on the electron temperature (Osterbrock \& Ferland 2006): $\epsilon(\lambda) \propto T_{\mathrm{e}}^{-1 / 2} \exp \left(-E_{\mathrm{ex}} / k T_{\mathrm{e}}\right)$, where $E_{\mathrm{ex}}$ is the excitation energy of the upper level of a CEL transition. We estimated that the errors in our [O III] temperatures typically introduce $\sim 9-12 \%$ uncertainties in the CEL ionic abundances.

\subsubsection{Elemental Abundances}

The total elemental abundances derived for the seven PNe are presented in Table 6, together with the Solar abundances from Asplund et al. (2009). The He/H elemental abundances of our targets were calculated by adding the $\mathrm{He}^{+} / \mathrm{H}^{+}$and $\mathrm{He}^{2+} / \mathrm{H}^{+}$ratios. Whenever available, the ionization correction factors (ICFs) given by Kingsburgh \& Barlow (1994) were used to calculate 

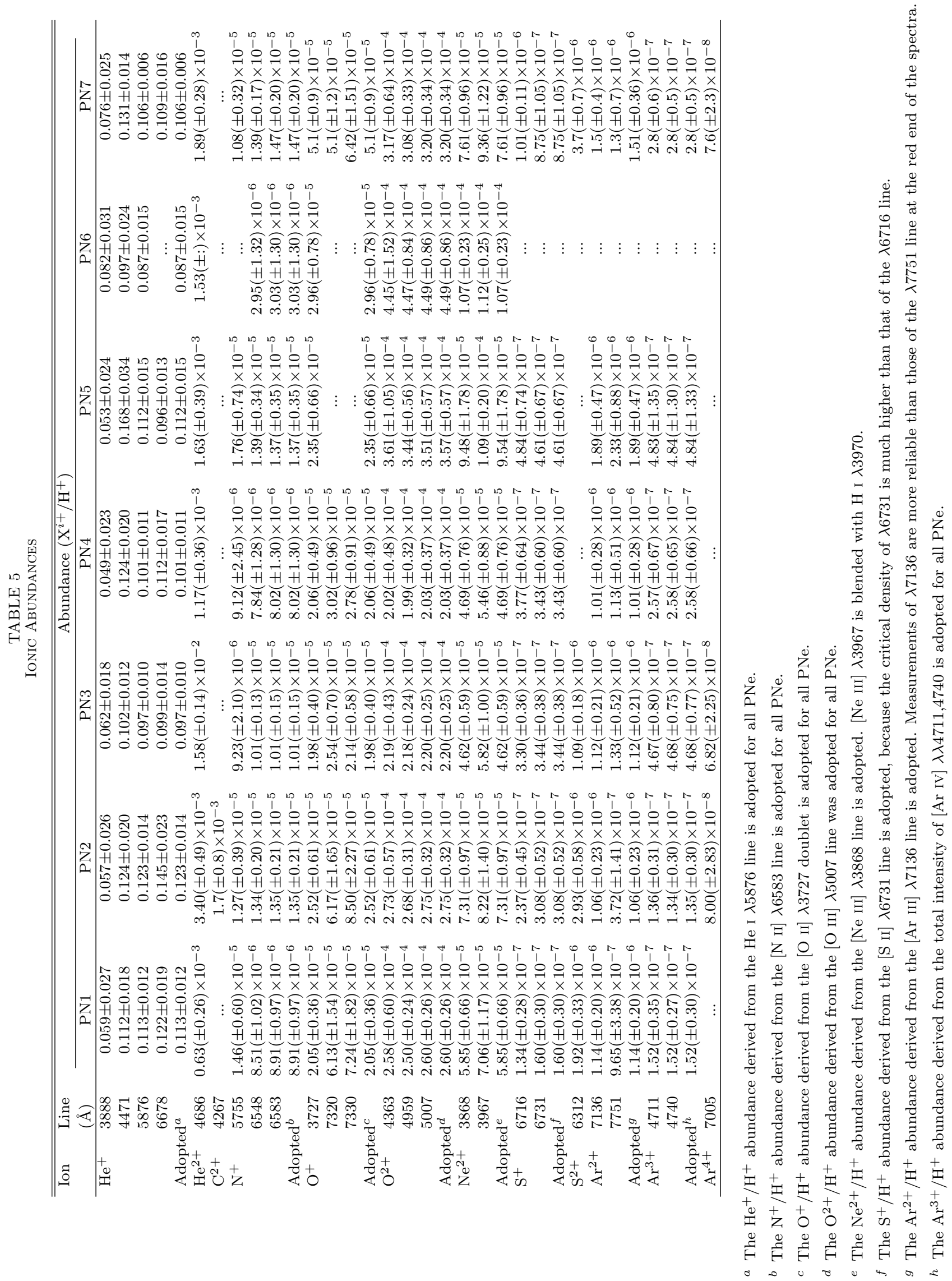
the total abundances of the heavy elements. The ICF values used to calculate the elemental abundances for the seven PNe are given in Table 7. It is worth mentioning that when calculating the elemental abundance of argon, depending on the detection or absence of the [Ar v] $\lambda 7005$ line, different ICFs have been used for our targets (Equations A30 and A34 in Kingsburgh \& Barlow 1994). The same scheme was adopted in the calculations of sulfur: The ICFs used for the targets, where the [S III] $\lambda 6312$ auroral line was detected, are different from those where the [S III] line was not detected (Equations 15 and A36 in Kingsburgh \& Barlow 1994).

Errors in the brackets following the total elemental abundances in Table 6 were estimated based on the errors in the ionic abundances through a simple propagation paradigm. For helium, the error is mainly contributed by uncertainties in the $\mathrm{He}^{+} / \mathrm{H}^{+}$ratio, whose concentration is much higher than that of $\mathrm{He}^{2+}$ (Table 5). For heavy elements, the errors can also be introduced by the use of ICFs. This source of error is negligible for oxygen, whose ICF is always close to unity (Table 7). For other heavy elements, uncertainties introduced by ICFs could be significant. Abundances of nitrogen and neon were derived based on the ionic and elemental abundances of oxygen, and thus are expected to be reliable. The total sulfur abundance is generally quite uncertain, as only the [S II] lines are well observed for this element. Although the [S III] $\lambda 6312$ line was also detected in the spectra of some PNe, it arises from an auroral transition $\left(3 \mathrm{p}^{2}{ }^{1} \mathrm{D}_{2}\right.$ $\left.-3 \mathrm{p}^{2}{ }^{1} \mathrm{~S}_{0}\right)$, hence it is particularly temperature sensitive. Measurements of the [S III] $\lambda 6312$ line is affected by the subtraction of the bright [O I] $\lambda 6300$ skyline. Besides, this [S III] line is also blended with the He II $\lambda 6311$ $\left(5 \mathrm{~g}^{2} \mathrm{G}-16 \mathrm{~h}^{2} \mathrm{H}^{\mathrm{o}}\right)$ line. Uncertainties and systematic errors in the ICFs are difficult to define, and thus they were not considered for the error estimate of our objects. The actual uncertainties in the elemental abundances of nitrogen, neon, sulfur and argon in Table 6 must be regarded as lower limits of the real abundance uncertainties.

\subsection{Duplication with Recent Spectroscopic Surveys}

Of all our targets, including the three PNe studied in Paper I, only PN4 (M77) was observed by Sanders et al. (2012), who obtained spectra for $253 \mathrm{H}$ II regions and 407 PNe in M31 using the Hectospec multi-fiber spectrograph attached to the $6.5 \mathrm{~m}$ MMT. Oxygen abundance was derived for $51 \mathrm{PNe}$ in the sample of Sanders et al. (2012) based on the electron temperatures estimated from the [O III] $\lambda 4363$ auroral line. However, the $\lambda 4363$ line was not detected in the MMT spectrum of M77, and thus the $\mathrm{O} / \mathrm{H}$ ratio was not estimated. For the most prominent nebular emission lines, fluxes measured in our GTC spectrum of M77 only differ slightly from those of the MMT spectrum: $2 \%$ for the [O III] $\lambda \lambda 4959,5007$ lines, $7 \%$ for the $[\mathrm{N}$ II $] \lambda \lambda 6548,6583$ lines, $12 \%$ for the [O II] $\lambda 3727$ lines, and $20-40 \%$ for the [S II] $\lambda \lambda 6716,6731$ lines.

\section{DISCUSSION}

\subsection{The Spatial and Kinematical Distribution}

Modern large-area surveys have revealed that M31 has an extended stellar disk and a huge halo $(\geq 300 \mathrm{kpc}$; Ibata et al. 2005, 2007, 2014). The outer regions of M31 are extremely complex. Numerous structures such as streams, loops, and overdensity regions were discovered throughout the halo (e.g., McConnachie et al. 2009, Lewis et al. 2013 : Ibata et al. 2014). These features, with the Northern Spur and the Giant Stream being the most prominent, are mostly associated with accretion/interaction of satellite dwarf galaxies. Although the possible connection between the Northern Spur and the Giant Stream had already been inferred by Ferguson et al. (2002) and McConnachie et al. (2003), Merrett et al. (2003) were the first to explicitly propose this connection. Based on a kinematic study of $\sim 20$ PNe in the disk of M31, Merrett et al. (2003) presented a possible orbit for the stellar stream in M31, which connects the Giant Stream to the Northern Spur. The Northern Spur is located at the turning point of this model orbit, which is strongly warped as the stellar stream passes nearby the center of M31 (Figure 7, upper-left panel).

Figure 7 shows the positions of PNe observed by Merrett et aI. (2006) in the $X-Y$ coordinate system in an M31-based reference frame, where $X$ lies along the major axis of M31 and increases toward the southwest, and $Y$ lies along the minor axis and increases toward the northwest. Both coordinates were calculated following the geometric transformations of Huchra et al. (1991). The orbit proposed by Merrett et al. (2003) is presented along with the PNe in Figure 7. The two side (bottom and right) panels in Figure 7 shows the projection of this stellar orbit in the line-of-sight velocity with respect to M31 $\left(v_{\text {los }}\right)$ versus distance along the major and minor axes. PNe in the Northern Spur and those associated with the Giant Stream are highlighted. The seven PNe in this study and the three observed in Paper I are also highlighted (see explanation of symbols in the caption of Figure 7).

The PNe in the two substructures are generally associated with the stellar orbit of Merrett et al. (2003), and this association is particularly consistent for those located on the Giant Stream. The three Northern Spur PNe observed in Paper I (see the discussion therein) are located at the turning point of the orbit. The positions of the seven objects targeted by the current work show excellent agreement with the orbit, both spatially and kinematically. The six PNe, named PN1-PN6, as carefully selected from the catalog of Merrett et al. (2003), were identified to be located in the two substructures by Merrett et al. (2006). It is worth noting that the spatial extent of the stellar orbit was originally confined to within $\sim 4^{\circ} \times 4^{\circ}$ centered on M31 (see Figure 2 of Merrett et al. 2003). The newly discovered PN7, with $v_{\text {los }}$ measured by the LAMOST spectroscopy, was located 3.65 from M31's center, beyond the orbit's extent of Merrett et al. (2003). However, the spatial position of PN7 matches well the extrapolation of the projected orbit in the tangential direction (the thick green dashed line in Figure 7).

Although slight deviation between PN7 and the extrapolated orbit exists in the $v_{\text {los }}$ vs. $X$ and $v_{\text {los }}$ vs. $Y$ diagrams (Figure 7, bottom and right panels), the agreement is still reasonable within the possible dispersion of the orbit, as Merrett et al. (2003) claimed that the stellar stream is probably not in reality a single orbit, but a family of adjacent orbits, and the singular nature of the potential of their simplified model also amplifies the dispersion in the orbit and increases the spread in the 
observed $v_{\text {los }}$ to $\sim 100 \mathrm{~km} \mathrm{~s}^{-1}$. This model orbit is only a generic representative of the stellar streams that connect the two photometric features.

The kinematics of the 27 outer-disk/halo PNe observed by Kwitter et al. (2012, Kwitter12; 16 PNe), Balick et al. (2013, Balick13; 2 PNe), and Corradi et al. (2015, Corradi15; $9 \mathrm{PNe}$ ) generally resemble the rotation pattern of the classical disk of M31 (Figure 7. bottom and right panels), and can be kinematically distinguished from our sample, which belong to the substructures and are mostly well located on the stellar stream of Merrett et al. (2003). Even for the PNe at very large radii, e.g., those along the major axis of M31 observed by Corradi et al. (2015), the kinematics still follow the extended disk. The kinematics of PNe in the Northern Spur (shown as open circles in Figure 7) are indistinguishable from those of the disk, although this substructure is located some distance from the plane.

\subsection{Abundance Correlations}

PNe probes the past chemical composition of the interstellar medium (ISM). Abundances of the $\alpha$-elements such as $\mathrm{O}, \mathrm{Ne}, \mathrm{S}$, and $\mathrm{Ar}$, of a $\mathrm{PN}$ represent the chemistry of the ISM in the era when the progenitor star just formed. On the other hand, H II regions trace the current abundances of the ISM. Giant extragalactic H II regions, where massive star formation activities occur, are amongst the most prominent features seen in a gas-rich, star-forming galaxy. They provide probes of relatively homogeneous interstellar material which generally show little evidence of abundance enhancements produced by the evolved stars embedded in them (e.g., Wofford 2009). A comparison of the abundances of PNe and $\mathrm{H}$ II regions at the same site (e.g., on the galaxy disk) is useful for studying the enrichment history of galaxies (e.g., Stanghellini et al. 2010). Investigation of relations between the abundances of different $\alpha$-elements helps to constrain the stellar evolution models and quantify the relative yields of each element in the asymptotic giant branch (AGB) stars, the immediate progenitors of PNe (e.g., Kwitter \& Henry 2001; Milingo et al. 2002, Kwitter et al. 2003, Henry et al. 2004). The latest review on the status of modeling the evolution and nucleosynthesis of AGB stars was presented by Herwig (2005).

Figures 812 show the abundance correlations of our sample as well as those for the M31 disk and bulge PNe from the literature. Overplotted in these figures are the M31 outer-disk PNe observed by Kwitter et al. (2012), Balick et al. (2013) and Corradi et al. (2015), as well as the M31 disk and bulge sample observed by Jacoby \& Ciardullo (1999). Also presented in the figures (except Figure 8) are the extragalactic $\mathrm{H}$ II region data, which set a baseline for the assumed tight linear behavior (in logarithmic scale) between different $\alpha$ elements: The M101 $\mathrm{H}$ II regions of Kennicutt et al. (2003, blue dots), the $\mathrm{H}$ II regions in low-metallicity blue compact galaxies observed by Izotov \& Thuan (1999) and Izotov et al. (2012, green dots), and the M31 $\mathrm{H}$ II regions observed by $\overline{\mathrm{Zu}}$ rita \& Bresolin (2012, magenta dots). These plots also include the solar values (Asplund et al. 2009) and the Orion nebular abundances (Esteban et al. 2004).

Figure 8 shows the $\mathrm{N} / \mathrm{O}$ vs. $\mathrm{He} / \mathrm{H}$ (left) and $\mathrm{N} / \mathrm{O}$ vs. $\mathrm{O} / \mathrm{H}$ (right) correlations. The Galactic disk PNe (mostly Type I) observed by Milingo et al. (2010) are also pre- sented in this figure for purpose of comparison. The $\mathrm{N} / \mathrm{O}$ and $\mathrm{He} / \mathrm{H}$ ratios of our sample are generally lower than those of the Galactic Type I PNe (Figure 8, left). Our $\mathrm{PNe}$ have $\mathrm{N} / \mathrm{O}$ ratios much lower than 0.8 , a criterion to distinguish the Galactic Type I and II PNe (Kingsburgh \& Barlow 1994). The seven PNe in this study, together with the three Northern Spur PNe in Paper I, are qualified to be classified as Type II, i.e., their progenitor stars have relatively low masses. The sample of Milingo et al. (2010) shows a positive correlation between N/O and $\mathrm{He} / \mathrm{H}$ and a decrease in $\mathrm{N} / \mathrm{O}$ with oxygen, indicating that more massive progenitors produce nitrogen at the expense of oxygen. Neither our targets nor the samples of Kwitter et al. (2012), Balick et al. (2013) and Corradi et al. (2015) show such trends as clearly as the Galactic PNe.

There is tight positive correlation between $\log (\mathrm{Ne} / \mathrm{H})$ and $\log (\mathrm{O} / \mathrm{H})$, as established based on the $\mathrm{H}$ II region data (e.g., Milingo et al. 2010). The neon-oxygen abundance distribution of our M31 PNe as well as those of Kwitter et al. (2012), Balick et al. (2013), and Corradi et al. (2015) generally agrees with this correlation within the errors, as shown in Figure 9 (left). Figure 9 (right) shows the flatness of $\mathrm{Ne} / \mathrm{O}$ in our sample. Observations of the Galactic PNe have revealed that destruction/production of the $\alpha$ elements, particularly in the case of neon, does occur (Milingo et al. 2010). Figure 9 shows that there is at lease one outlier in the sample of Jacoby \& Ciardullo (1999), indicating possible destruction of Ne. However, the uncertainties of Jacoby \& Ciardullo (1999) were not presented. Argon of our sample is generally correlated with oxygen, following the pattern of H II regions; however, the M31 outer-disk PNe of Kwitter et al. (2012), Balick et al. (2013), and Corradi et al. (2015) have systematically lower argon abundances (Figure 10. The exact cause of this systematic difference in the argon abundances is unclear, although the use of different ICFs could not be totally ruled out. The sample of Jacoby \& Ciardullo (1999), as well as the Type I PNe of Milingo et al. (2010), have large scatter.

The sulfur anomaly, first noticed in Galactic PNe by Henry et al. (2004) and confirmed by Milingo et al. $(2010)$, is also present in the M31 sample (Figure 11): The sulfur abundances of the M31 PNe are significantly lower than those of $\mathrm{H}$ II regions at similar oxygen abundance. Besides, the scatter in the PN sulfur abundances for a given metallicity is much larger than that in $\mathrm{H}$ II regions. The sulfur anomaly is also evident in the diagrams of $\mathrm{S} / \mathrm{H}$ vs. $\mathrm{Ne} / \mathrm{H}$ (Figure 12 , left) and $\mathrm{S} / \mathrm{H}$ vs. $\mathrm{Ar} / \mathrm{H}$ (Figure 12, right). Henry et al. (2004) originally proposed that the apparently low sulfur abundances were likely due to ICF problems. This argument was later confirmed by Henry et al. (2012). Under the physical conditions of PNe, a significant amount of sulfur is in the triply ionized stage $\mathrm{S}^{3+}$, which can not be observed in the optical. The presence of $\mathrm{S}^{3+}$ is also indicated by the detection of $\mathrm{He}^{2+}$ (e.g., the strong He II $\lambda 4686$ line of PN3), because the ionization potential of $\mathrm{He}^{+}(54.4 \mathrm{eV})$ is higher than that of $\mathrm{S}^{2+}(34.8 \mathrm{eV})$. However, Milingo et al. (2010, also Henry et al. 2012) found that the sulfur anomaly persists even after including the $\mathrm{S}^{3+} / \mathrm{H}^{+}$ abundances derived from the infrared ISO data. It has been suggested that the sulfur anomaly could be due to the formation of dust such as MgS and FeS (Pottasch 
TABLE 6

Elemental ABUndances ${ }^{a}$

\begin{tabular}{|c|c|c|c|c|c|c|c|c|}
\hline \multirow[t]{2}{*}{ Elem. } & \multicolumn{8}{|c|}{$\overline{\mathrm{X} / \mathrm{H}}$} \\
\hline & \multicolumn{2}{|l|}{ PN1 } & \multicolumn{2}{|l|}{$\mathrm{PN} 2$} & \multicolumn{2}{|l|}{ PN3 } & \multicolumn{2}{|l|}{ PN4 } \\
\hline $\mathrm{He}$ & $0.113 \pm 0.013$ & 11.05 & $0.126 \pm 0.017$ & 11.10 & $0.113 \pm 0.014$ & 11.05 & $0.102 \pm 0.012$ & 11.01 \\
\hline $\mathrm{C}$ & $\ldots$ & $\ldots$ & $2.15( \pm 0.73) \times 10^{-3}$ & 9.33 & $\ldots$ & $\ldots$ & $\ldots$ & $\ldots$ \\
\hline $\mathrm{N}$ & $4.39( \pm 1.10) \times 10^{-5}$ & 7.64 & $6.60( \pm 2.77) \times 10^{-5}$ & 7.82 & $1.15( \pm 0.50) \times 10^{-4}$ & 8.06 & $6.44( \pm 2.83) \times 10^{-5}$ & 7.81 \\
\hline $\mathrm{O}$ & $2.81( \pm 0.93) \times 10^{-4}$ & 8.45 & $3.06( \pm 0.95) \times 10^{-4}$ & 8.49 & $2.69( \pm 0.57) \times 10^{-4}$ & 8.43 & $2.34( \pm 0.77) \times 10^{-4}$ & 8.37 \\
\hline $\mathrm{Ne}$ & $7.36( \pm 1.40) \times 10^{-5}$ & 7.87 & $9.39( \pm 3.94) \times 10^{-5}$ & 7.97 & $5.65( \pm 2.54) \times 10^{-5}$ & 7.75 & $5.40( \pm 2.38) \times 10^{-5}$ & 7.73 \\
\hline S & $1.52( \pm 0.68) \times 10^{-6}$ & 6.18 & $2.90( \pm 1.30) \times 10^{-6}$ & 6.46 & $4.60( \pm 2.53) \times 10^{-6}$ & 6.66 & $3.98( \pm 2.23) \times 10^{-6}$ & 6.60 \\
\hline $\mathrm{Ar}$ & $1.62( \pm 0.73) \times 10^{-6}$ & 6.21 & $1.60( \pm 0.75) \times 10^{-6}$ & 6.20 & $1.82( \pm 0.80) \times 10^{-6}$ & 6.26 & $1.44( \pm 0.76) \times 10^{-6}$ & 6.16 \\
\hline \multirow[t]{2}{*}{ Elem. } & \multicolumn{8}{|c|}{$\mathrm{X} / \mathrm{H}$} \\
\hline & PN5 & & PN6 & & PN7 & & Solar $^{b}$ & \\
\hline $\mathrm{He}$ & $0.114 \pm 0.015$ & 11.05 & $0.089 \pm 0.015$ & 10.95 & $0.108 \pm 0.012$ & 11.03 & 0.085 & 10.93 \\
\hline $\mathrm{C}$ & $\ldots$ & $\ldots$ & $\ldots$ & $\ldots$ & $\ldots$ & $\ldots$ & $2.69 \times 10^{-4}$ & 8.43 \\
\hline $\mathrm{N}$ & $1.13( \pm 0.43) \times 10^{-4}$ & 8.05 & $4.96( \pm 1.73) \times 10^{-5}$ & 7.70 & $9.84( \pm 2.75) \times 10^{-5}$ & 7.99 & $6.76 \times 10^{-5}$ & 7.83 \\
\hline $\mathrm{O}$ & $4.11( \pm 1.20) \times 10^{-4}$ & 8.61 & $4.84( \pm 1.31) \times 10^{-4}$ & 8.68 & $3.81( \pm 0.96) \times 10^{-4}$ & 8.58 & $4.89 \times 10^{-4}$ & 8.69 \\
\hline $\mathrm{Ne}$ & $1.10( \pm 0.48) \times 10^{-4}$ & 8.04 & $1.16( \pm 0.48) \times 10^{-4}$ & 8.06 & $9.07( \pm 3.53) \times 10^{-5}$ & 7.96 & $8.51 \times 10^{-5}$ & 7.93 \\
\hline $\mathrm{S}$ & $1.07( \pm 0.61) \times 10^{-5}$ & 7.03 & $\ldots$ & $\ldots$ & $9.38( \pm 4.13) \times 10^{-6}$ & 6.97 & $1.32 \times 10^{-5}$ & 7.12 \\
\hline Ar & $2.71( \pm 1.30) \times 10^{-6}$ & 6.43 & $\ldots$ & $\ldots$ & $2.20( \pm 0.83) \times 10^{-6}$ & 6.34 & $2.51 \times 10^{-6}$ & 6.40 \\
\hline
\end{tabular}

$a$ For each PN, the abundances in the left column are linear and those in the right column are logarithm, $\log (\mathrm{X} / \mathrm{H})+12$.

$b$ Asp]und et al. (2009).

TABLE 7

IONIZATION CORRECTION FACTORS

\begin{tabular}{lrrrrrrr}
\hline \hline Elem. & \multicolumn{7}{c}{ ICF } \\
\cline { 2 - 8 } & PN1 & PN2 & PN3 & PN4 & PN5 & PN6 & PN7 \\
\hline $\mathrm{He}$ & 1.000 & 1.000 & 1.000 & 1.000 & 1.000 & 1.000 & 1.000 \\
$\mathrm{C}$ & $\ldots$ & 1.285 & $\ldots$ & $\ldots$ & $\ldots$ & $\ldots$ & $\ldots$ \\
$\mathrm{N}$ & 4.932 & 4.900 & 11.412 & 8.029 & 8.218 & 16.354 & 6.705 \\
$\mathrm{O}$ & 1.002 & 1.018 & 1.105 & 1.008 & 1.009 & 1.011 & 1.011 \\
$\mathrm{Ne}$ & 1.258 & 1.285 & 1.224 & 1.152 & 1.151 & 1.078 & 1.191 \\
$\mathrm{~S}$ & 1.266 & 1.263 & 1.608 & 1.448 & 1.458 & 1.796 & 1.375 \\
$\mathrm{Ar}$ & 1.254 & 1.256 & 1.096 & 1.142 & 1.138 & 1.065 & 1.175 \\
\hline
\end{tabular}

\& Bernard-Salas 2006), but the exact amount of sulfur deficit caused by this mechanism is difficult to estimate.

\subsection{Stellar Population}

The $\mathrm{He} / \mathrm{H}$ and $\mathrm{N} / \mathrm{O}$ ratios as well as the abundance ratios of the $\alpha$-elements of our PNe generally span in the ranges within those of the disk PNe observed by Kwitter et al. (2012), Balick et al. (2013) and Corradi et al. (2015). This indicates that our sample have probably similar properties as those of Kwitter et al. (2012). Adopting the linear least-squares fit to the extinction parameter $c(\mathrm{H} \beta)$ versus $\mathrm{PN}$ core-mass relation presented by Kwitter et al. (2012, Figure 4), we estimate that the core masses of our sample are in the range $\sim 0.59-0.65 M_{\odot}$. The extinction versus core mass plot of Kwitter et al. (2012) seems to indicate that this relation is insensitive to core mass below $\sim 0.59 M_{\odot}$ when the extinction parameter $c(\mathrm{H} \beta) \lesssim 0.20$. Five object in our sample are in this range. Here our extinction parameters $c(\mathrm{H} \beta)$ have been corrected for the Galactic average foreground extinction toward M31 $(E(B-V)=0.062$; Schlegel et al. 1998), assuming the ratio $c(\mathrm{H} \beta) / E(B-\bar{V})=1.4$ (Kaler \& Lutz 1985). The initial masses of our target PNe are in the range $1.81-2.26 M_{\odot}$, as estimated using the initial-final mass relationship of white dwarfs given by Catalán et al. (2008, Equation 1). The progenitors' main-sequence lifetimes of our sample $\mathrm{PNe}$ are in the range $0.85-1.8 \mathrm{Gyr}$, which was estimated using the stellar evolution model results of Schaller et al. (1992, Table 45). Although the model tracks of Vassiliadis \& Wood (1994) for the post-AGB evolution were not used to constrain the core masses of our target $\mathrm{PNe}$, the range of our core masses (also the initial masses) may be narrower than those of the sample studied by Kwitter et al. (2012).

Our estimate of the PN core masses is not robust, although the extinction versus PN core mass correlation presented in Kwitter et al. (2012) can be physically explained, and has already been validated by Jacoby \& Ciardullo (1999) and discussed in more detail in Ciardullo \& Jacoby (1999). This correlation is a simple consequence of the greater mass loss and faster evolution times of relatively high-mass stars (Ciardullo \& Jacoby 1999). The mass-loss processes through the AGB wind are complex, and the circumstellar extinction depends largely on these ejection processes as well as on the central star evolution. Thus the linear fit of Kwitter et al. (2012) may still be crude, and it is inappropriate to simply use this relation to derive the core masses. On this regard, our core masses are of large uncertainty. With the future availability of deep GTC spectra obtained for more PNe in the substructures of $\mathrm{M} 31^{6}$, we will construct detailed photoionization models for our PN sample, and more robust core masses will be derived. This will be presented in a separate paper.

Previous deep Hubble Space Telescope Advanced Camera for Surveys (ACS) imaging revealed that the stellar fields at the Giant Stream are older than those on the disk (Bernard et al. 2015), suggestive of an early-type progenitor. The stream fields are generally consistent with the direction of target PN7 (Figure 1). The stellar field of the Northern Spur targeted by Bernard et al. (2015) also well coincides with the position of target PN3 (Figure 1). In all ACS fields studied, Bernard et al.

6 We have recently been awarded more observing time at the $10.4 \mathrm{~m}$ GTC in Semester 2015B (September 12015 - February 29 $2016)$ to carry out deep spectroscopy of 11 more PNe in the substructures of M31 (GTC program \#GTC92-15B, PI: X. Fang). 


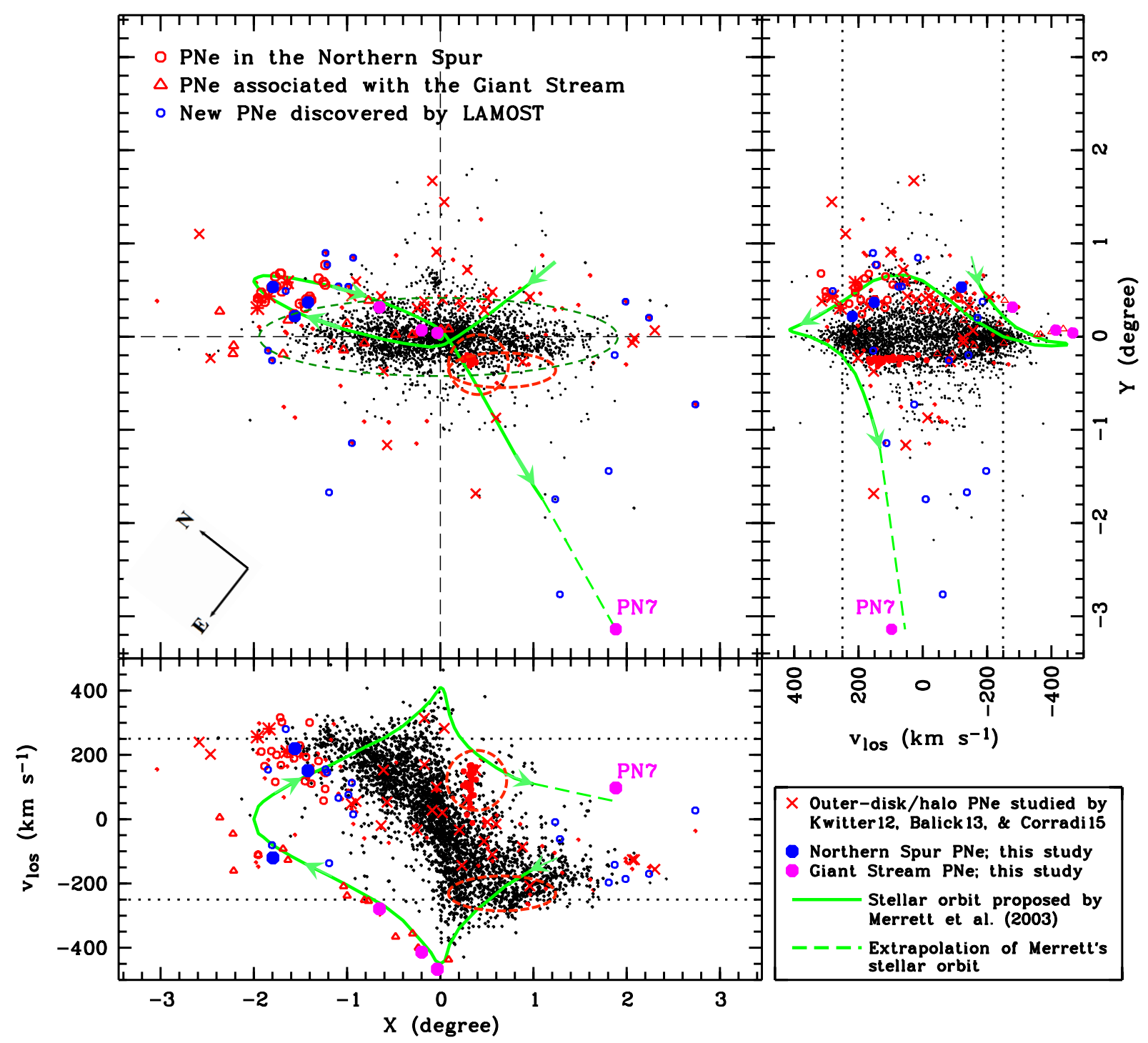

FIG. 7.- Spatial distribution and kinematics of PNe in M31 (see the description of the $X$ and $Y$ coordinates in the text). PNe samples are from Merrett et al. (2006, black dots; including the sample of Hurley-Keller et al. 2004), Kniazev et al. (2014, red dots), and the LAMOST survey (Yuan et al. 2010 blue open circles). Red open circles represent the Northern Spur PNe and red open triangles are those associated with the Giant Stream. The three Northern Spur PNe studied in Paper I are the red asterisks. The seven PNe in this study are highlighted by the blue and magenta filled circles (see the bottom-right legend, also Figure 1), and PN7, a distant PN newly identified by LAMOST (see Section 2.1), is marked. Red crosses are the outer-disk and halo PNe observed by Kwitter et al. (2012), Balick et al. (2013), and Corradi et al. (2015). In the upper left panel: overplotted is the stellar orbit (thick green curve) proposed by Merrett et al. (2003); the green dashed elhpse has a semimajor axis of $2^{\circ}(\sim 27 \mathrm{kpc})$ and represents a disk with an inclination angle $i=77.7$ (de Vaucouleurs 1958). In the two side panels (bottom and right): a projection of the orbit in line-of-sight velocity with respect to M31, $v$ los, vs. distance along the major and minor axes of M31 is superimposed on the PNe data. All velocities of PNe are corrected for the systemic velocity $-306 \mathrm{~km} \mathrm{~s}^{-1}$ (Corbelli et al. 2010). Arrows on the orbit show the direction along the stream. The red dashed circle and ellipse show the locations of satellites M32 and And VIII, respectively, and the red dots in the red dashed circle are the PNe belonging to M32. The thick green dashed lines are tangential extrapolations of the stellar orbit. The black dotted lines at $\pm 250 \mathrm{~km} \mathrm{~s}^{-1}$ (Corbelli et al. 2010) show the adopted circular speed of M31 disk. The format of the figure generally follows Figure 2 of Merrett et al. (2003 also Figure 17 in Paper I) and the stellar orbit was reproduced based on that figure (with permission of the authors).

(2015) found a significant burst of star formation 2 Gyr ago, in rough agreement with the main-sequence ages we estimated for our target PNe.

\subsection{Radial Distribution of Oxygen}

The radial distribution of the oxygen abundances of our sample (the red open and filled circles) is presented in Figure 13 where we also plot the M31 outer-disk/halo samples observed by Kwitter et al. (2012, black filled circles), Balick et al. (2013) and Corradi et al. (2015, black filled diamonds). The PN samples of Sanders et al. (2012, black open circles) and Jacoby \& Ciardullo (1999 black open triangles) are also plotted. The M31 H II regions observed by Zurita \& Bresolin (2012) and Esteban et al. (2009) are also presented in the figure for purpose of comparison. Galactocentric distances (in kpc) have been rectified for the effects of projection on the plane of sky assuming that all objects are located on the disk, except PN7, which is located on the extension of the Giant Stream and whose distance to the center of M31 is probably $\geq 100 \mathrm{kpc}$ if considering the three-dimensional structure of the Giant Stream (McConnachie et al.|2003). 

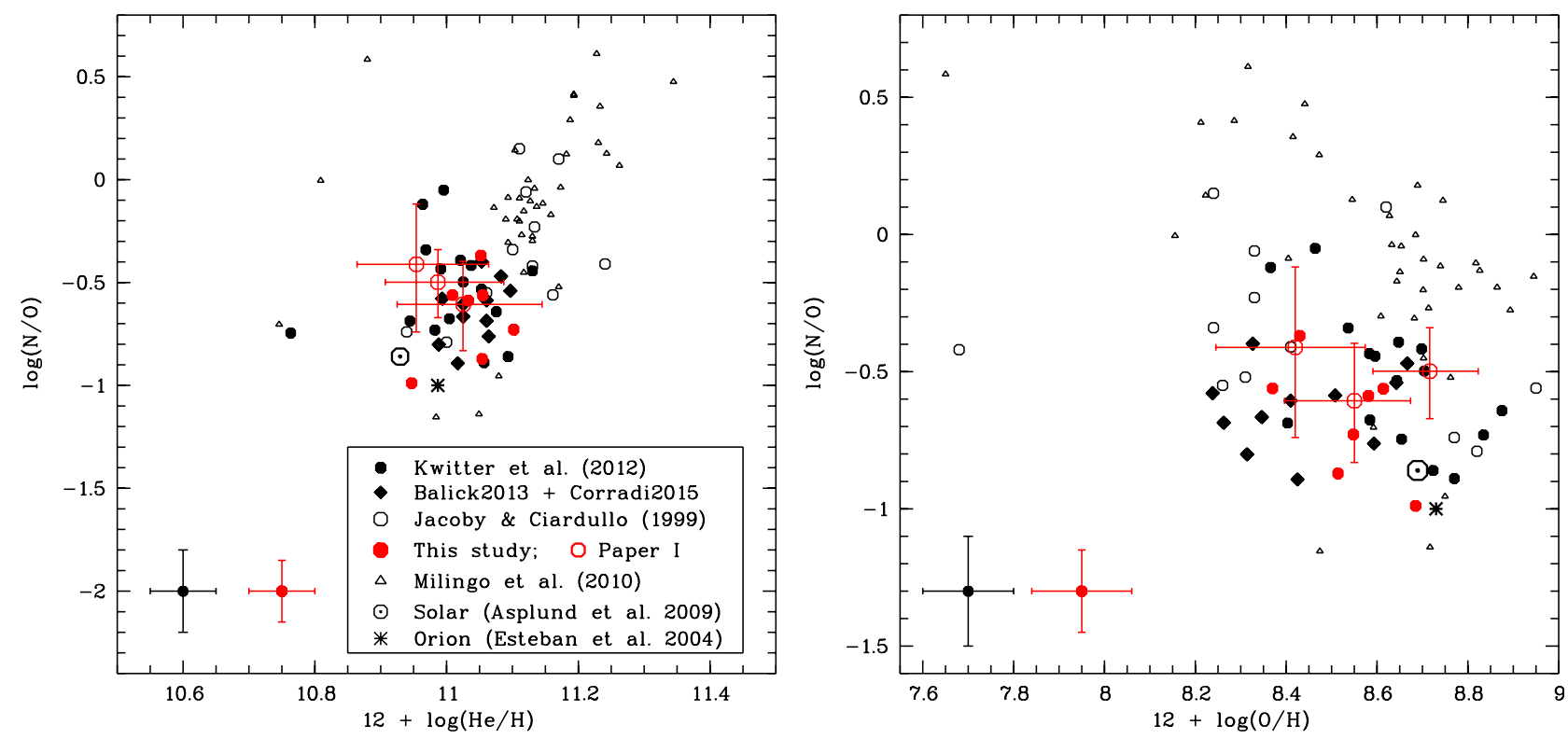

Fig. 8. - Left: $\log (\mathrm{N} / \mathrm{O})$ vs. $12+\log (\mathrm{He} / \mathrm{H})$. Right: $\log (\mathrm{N} / \mathrm{O})$ vs. $12+\log (\mathrm{O} / \mathrm{H})$. Different symbols represent different PNe samples (see legend). Explanation of the data sets is given in the text. Red open circles (along with error bars) are the three Northern Spur PNe observed in Paper I. Representative error bars of our targets and those of Kwitter et al. (2012, also Balick et al. 2013 and Corradi et al. 2015) are given in the lower-left corner. The symbols in Figures 912 have the same meaning.
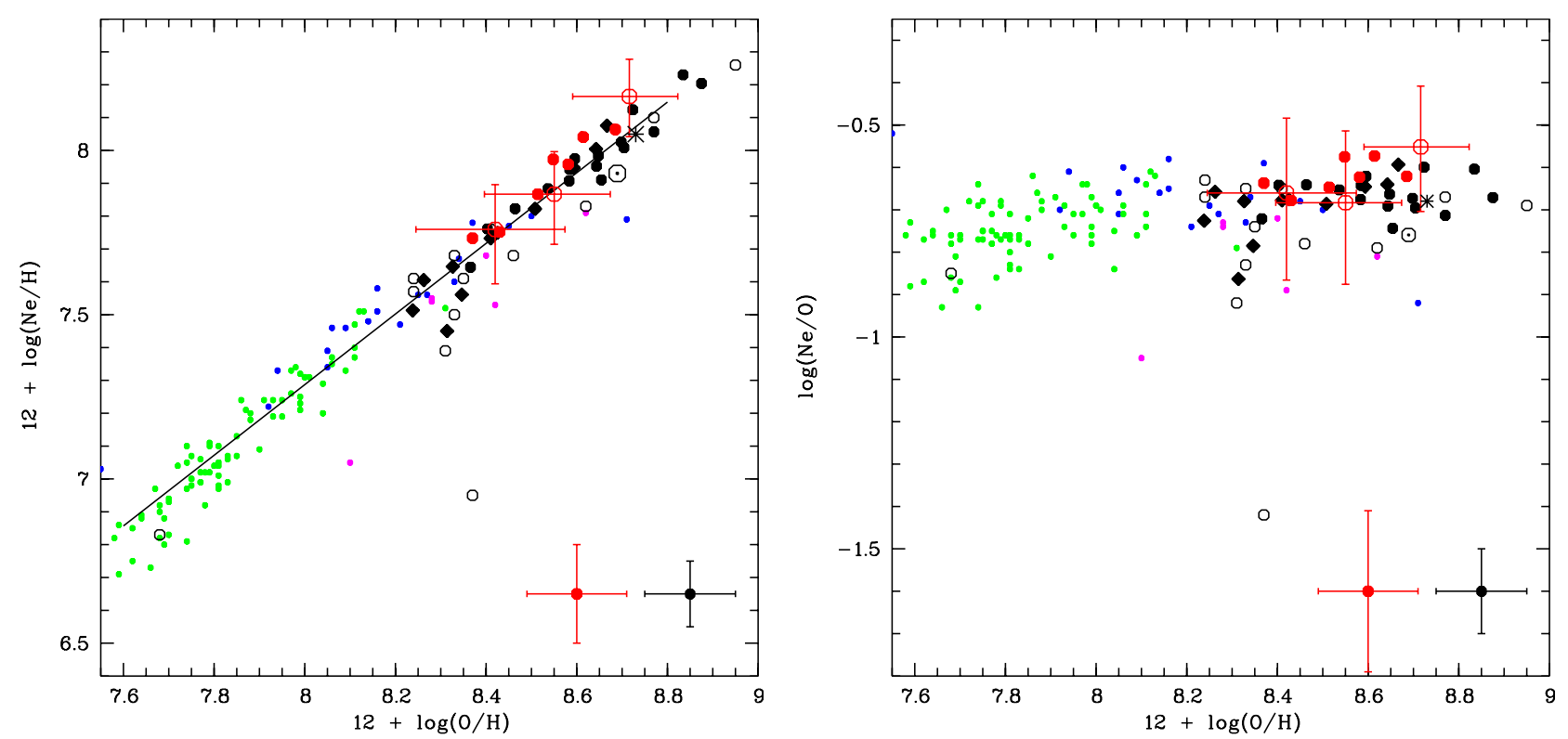

FIG. 9. - Left: $12+\log (\mathrm{Ne} / \mathrm{H})$ vs. $12+\log (\mathrm{O} / \mathrm{H})$. The solid black line is a least-squares linear fit to the $\mathrm{H}$ II region data. Right: $\log (\mathrm{Ne} / \mathrm{O})$ vs. $12+\log (\mathrm{O} / \mathrm{H})$. The small colored (green, blue and magenta) dots are $\mathrm{H}$ II regions and metal-poor blue compact galaxies (see references in the text). The symbols in Figures 1012 have the same meaning.

We assumed an inclination angle of $77^{\circ} .7$ for the M31 disk and a position angle of $37^{\circ} .7$ for the M31 main axis (de Vaucouleurs 1958) in the projection rectification.

The M31 disk sample observed by Kwitter et al. (2012) yield an oxygen gradient of $-0.011 \pm 0.004 \mathrm{dex} \mathrm{kpc}{ }^{-1}$ the target PN16, whose PN ID in Merrett et al. (2006) is 1074, was not included in gradient fit because of absence of the [O $\mathrm{II}] \lambda 3727$ line in the spectrum]. The two distant (halo) PNe observed by Balick et al. (2013) have the oxygen abundances comparable to that of the sun (Figure 13), indicating a possible flattening in the outer disk. Our sample, including the three Northern Spur PNe in
Paper I, spans a wide range in galactocentric distances and have rather homogeneous oxygen abundances. The oxygen abundance of PN7, the most distant PN in our sample, is consistent with those of the two outer-disk PNe of Balick et al. (2013) within the uncertainties. However, they belong to different groups: PN7 is associated with the Giant Stream both spatially and kinematically, while the kinematics of Balick's sample follow the rotation pattern of the classical disk of M31 (Figure 7). PN5 and PN6 are close to the galaxy center and both have oxygen abundances close to that of PN7. These three PNe are well associated with the extension of the Giant Stream 

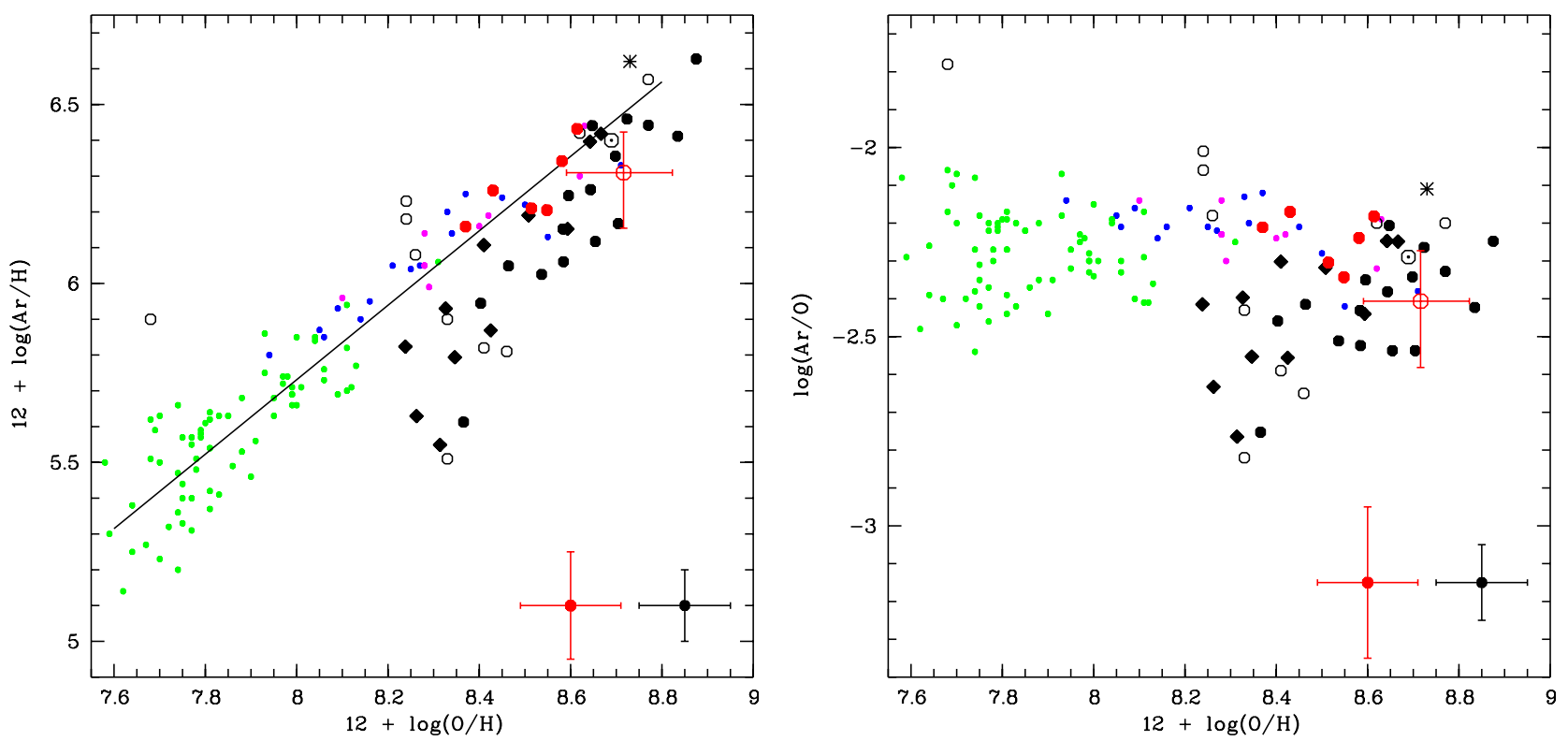

FIG. 10. - Same as Figure 9 but for $12+\log (\mathrm{Ar} / \mathrm{H})$ vs. $12+\log (\mathrm{O} / \mathrm{H})(\mathrm{left})$ and $\log (\mathrm{Ar} / \mathrm{O})$ vs. $12+\log (\mathrm{O} / \mathrm{H})($ right $)$.
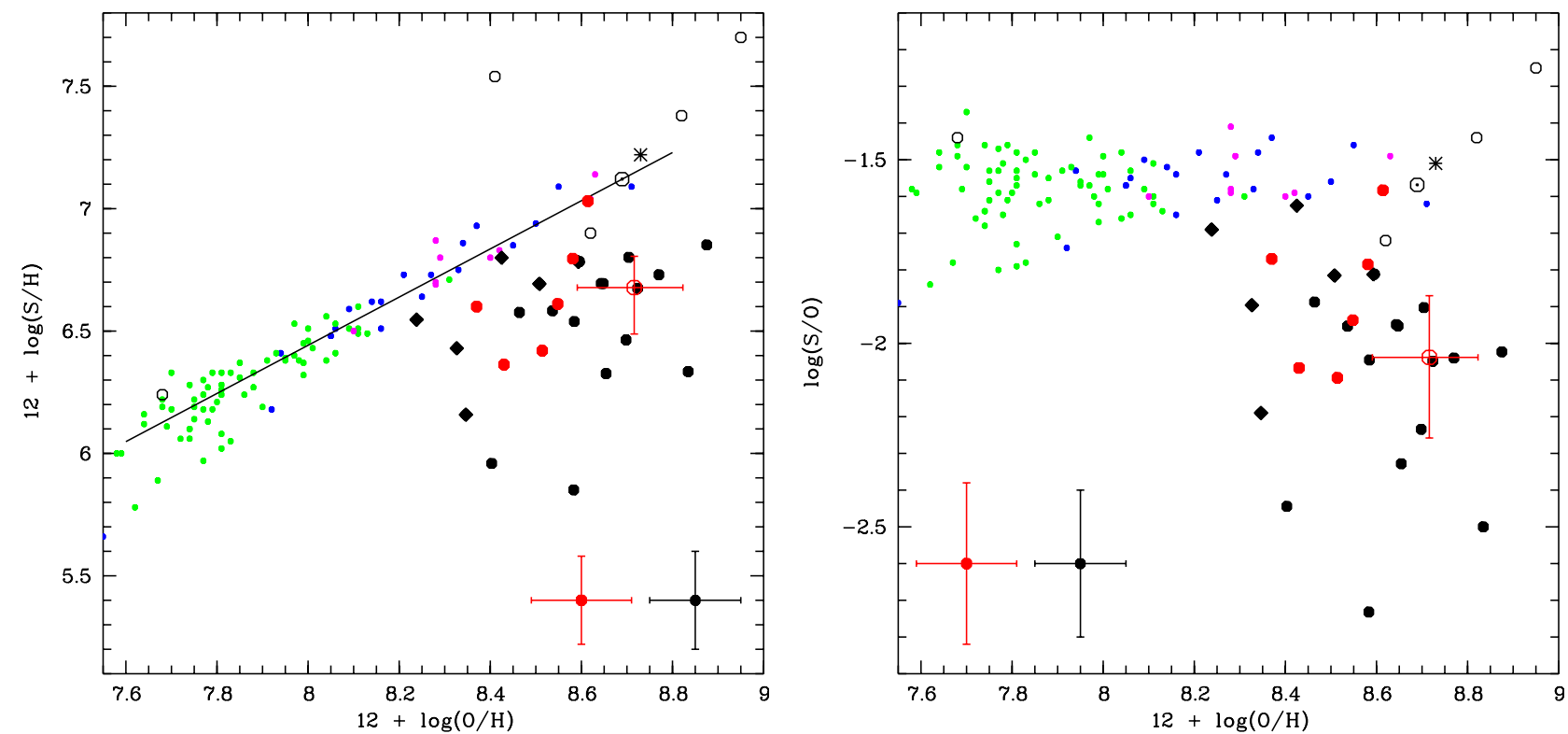

FIG. 11. - Same as Figure 9 but for $12+\log (\mathrm{S} / \mathrm{H})$ vs. $12+\log (\mathrm{O} / \mathrm{H})(\mathrm{left})$ and $\log (\mathrm{S} / \mathrm{O})$ vs. $12+\log (\mathrm{O} / \mathrm{H})($ right $)$.

of M31 (Figure 7).

PN1, PN2 and PN3 are located in the Northern Spur and their oxygen abundances are consistent with those in Paper I, which belong to the same substructure. The average $12+\log (\mathrm{O} / \mathrm{H})$ of our sample $(10 \mathrm{PNe})$ is $8.56 \pm 0.10$, about 0.1 dex below the solar value (8.69, Asplund et al. 2009), while the mean value of the 18 outer-disk PNe of Kwitter et al. (2012) and Balick et al. (2013) is $8.62 \pm 0.14$, which is consistent with ours within dispersion. The $\mathrm{O} / \mathrm{H}$ ratios of our ten $\mathrm{PNe}$ are also generally consistent within the errors. It is also worth noting that PN5 and PN6 have the oxygen abundances higher than the PN samples of Jacoby \& Ciardullo (1999) and Sanders et al. (2012) at similar galactocentric distances, although the latter two both show large scatter. The $\mathrm{O} / \mathrm{H}$ ratios of these two $\mathrm{PNe}$ are also higher than those of the M31 H II regions observed by Esteban et al. (2009) and Zurita \& Bresolin (2012).

\subsection{Possible Origin of the Substructure}

The carefully selected targets in this work, three belong to the Northern Spur and four associated with the Giant Stream substructure, have homogeneous oxygen abundances. Although they can not be clearly distinguished from the outer-disk sample of Balick et al. (2013) by the abundances, their kinematics generally follow the stellar orbit that connects the Northern Spur and the Giant Stream. The most intriguing object in our sam- 

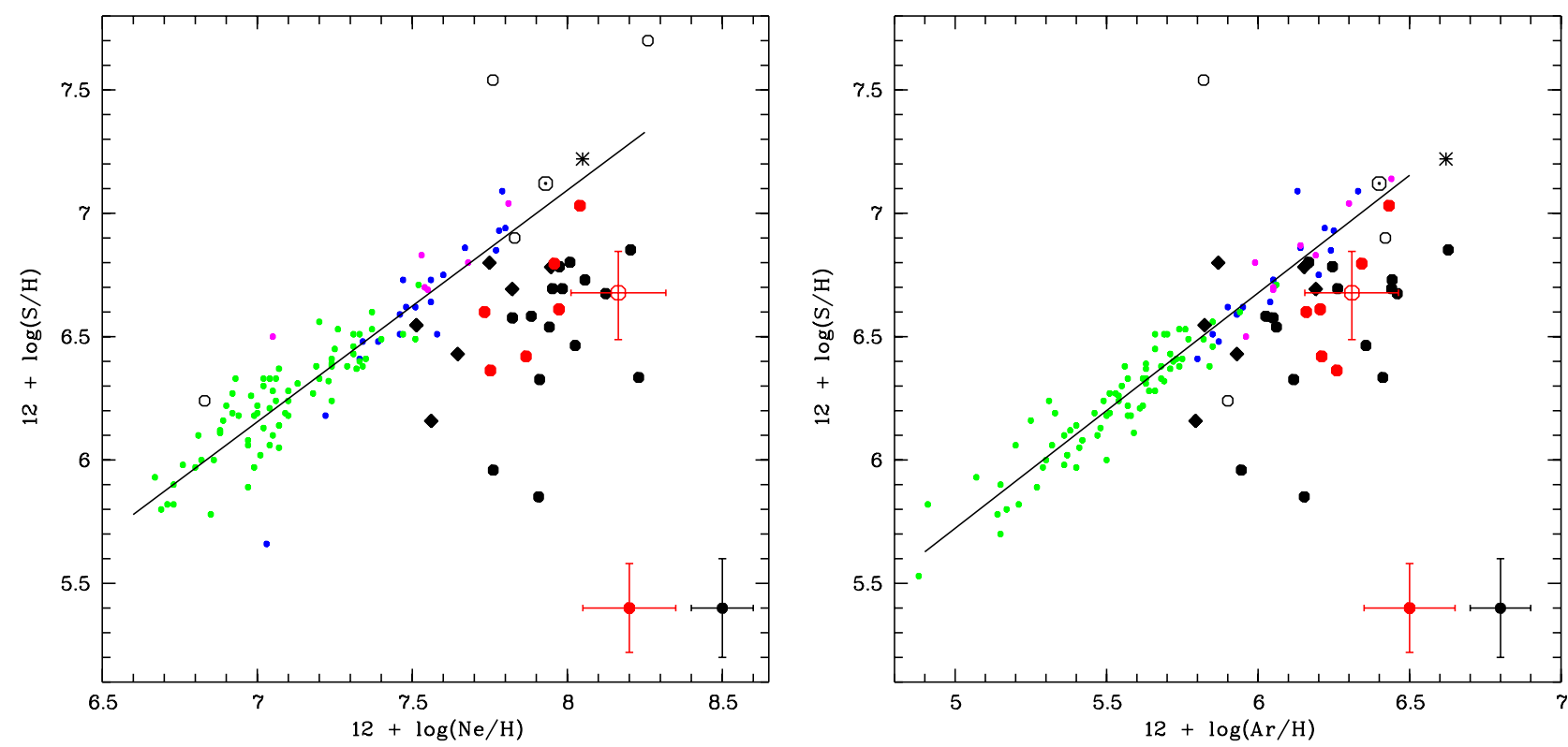

FIG. 12. - Same as Figure 9 but for $12+\log (\mathrm{S} / \mathrm{H})$ vs. $12+\log (\mathrm{Ne} / \mathrm{H})(\mathrm{left})$ and $12+\log (\mathrm{S} / \mathrm{H})$ vs. $12+\log (\mathrm{Ar} / \mathrm{H})(\mathrm{right})$.

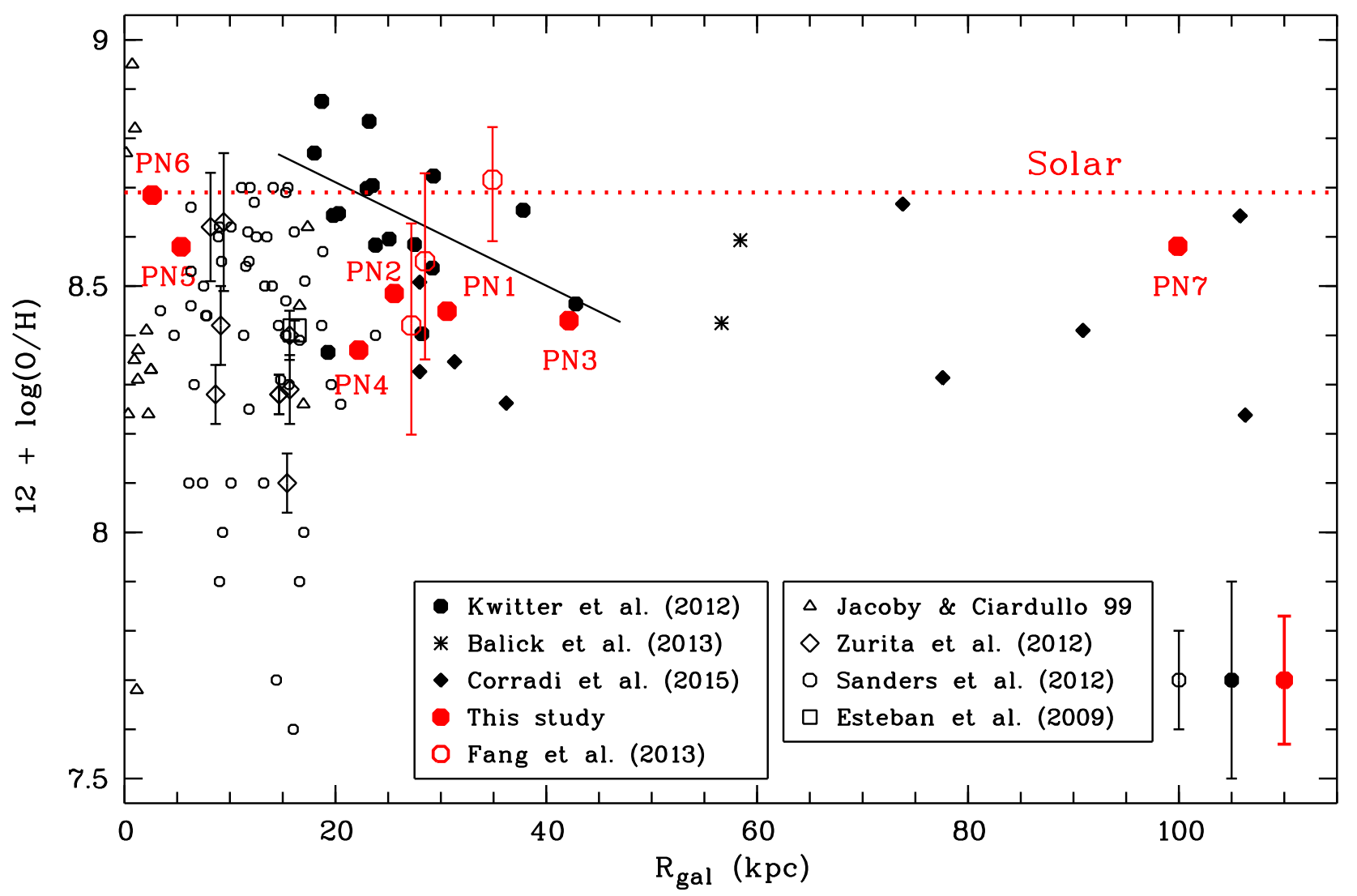

FIG. 13. - Distribution of oxygen abundances of PNe and H II regions in M31. The black filled circles are the 16 M31 disk PNe observed by Kwitter et al. (2012); black asterisks are the two outer-disk PNe observed by Balick et al. (2013); black filled diamonds are the nine outskirt PNe observed by Corradi et al. (2015). The black open circles are the PNe sample of Sanders et al. (2012); black open triangles are the sample from Jacoby \& Ciardullo (1999). Open diamonds are the nine disk H II regions analyzed by Zurita \& Bresolin (2012). The open square is an M31 H II region (K932) studied by Esteban et al. (2009). Red open circles are the three Northern Spur PNe in Paper I, and red filled circles are the seven PNe in this study. Galactocentric distances have been rectified. The actual position of PN7 is $\sim 100 \mathrm{kpc}$, as derived based on the three-dimensional structure of the Giant Stream (McConnachie et al. 2003). Representative errors of our sample as well as those of Kwitter et al. (2012, also Balick et al. 2013 and Corradi et al. 2015) and Sanders et al. (2012) are indicated in the lower right corner. The black straight line is a linear fit to the disk sample of Kwitter et al. (2012). The horizontal red dotted line is the solar oxygen abundance (Asplund et al. 2009). 
ple is PN7, a faint PN in the extension of the Giant Stream with a sky-projected angular distance of 3.65 . This PN is located probably $>100 \mathrm{kpc}$ from the center of M31, according to the three-dimensional structure of Giant Stream (McConnachie et al. 2003). The similarity in its oxygen abundance with those of PN5 and PN6, which are both close to the bulge of M31 (Figure 13), confirms that these three Stream PNe probably originated from the same stellar population. The spatial, kinematical and chemical information points to the possibility that these PNe have the same origin, and provides observational support for the stellar orbit, which was proposed by Merrett et al. (2003) based on the kinematic study of $\sim 20$ PNe spatially located on the M31 disk.

Ibata et al. (2001b) initially suggested that either or both of M32 and NGC 205 could be responsible for the Giant Stream, based on the geometrical alignment of the two satellites. The panoramic survey of RGB stars in the M31 halo by Ferguson et al. (2002) reveals that M32 seems to be a more promising candidate for the origin of the stream. These authors also inferred a possible connection between the Giant Stream and the extent of the Northern Spur, and even pointed out a strong warp of this connection orbit (if it does exist) near M31's nucleus. Based on a kinematic study of $\sim 20$ disk PNe, Merrett et al. (2003) constructed a model orbit that connects the Northern Spur and the Giant Stream, and proposed that the two substructures are the tidal tails (one is the leading part, and the other the trailing part) of M32, given that this dwarf elliptical is close to the orbit both in position (projected on the $X-Y$ plane) and velocity. Merrett et al. (2003) also provided two extra arguments to support this hypothesis: 1) the RGB in the Northern Spur and the Giant Stream are both particularly red compared with the rest of the halo of M31 (Ferguson et al. 2002), and 2) M32 appears to be highly tidally stripped. However, the exact position of M32 with respect to the disk of M31 remains yet to be determined (Mateo 1998).

The galaxy And VIII is a tidally disrupted satellite of M31, and it was located very close to M32 in the $X-Y$ plane (Figure 7) and might also be a candidate for the origin of the stellar stream (Merrett et al. 2003). This satellite was found to be strongly distorted, with a length of $\sim 10 \mathrm{kpc}$ and a width of a few kiloparsecs, and contains 5-12 PNe and has a systematic velocity of $-204 \mathrm{~km} \mathrm{~s}^{-1}$ with respect to M31 (Morrison et al. 2003). And VIII occupies the position where the Giant Stream meets the disk of M31, and the PNe in this satellite kinematically coincide with the model orbit (Figure 7, bottom). However, the kinematics of And VIII is opposite to that of M32, casting doubts on its possible origin of the stellar stream. Given the large scatter of the stellar stream both in positions and line-of-sight velocities, as discussed in Merrett et al. (2003), M32 is associated with the orbit. Although spectroscopic observations of PNe in M32 has been carried out (Richer et al.1999), accurate abundance determination is scarce. Future spectroscopic study of M32 PNe using the $8-10 \mathrm{~m}$ class telescopes will help to confirm their true nature.

\section{SUMMARY AND CONCLUSION}

In order to investigate the possible origin of substructures of M31 using PNe as a tracer of chemical abundances, we carried out deep spectroscopy of PNe kine- matically associated with the two most prominent substructures of M31, the Northern Spur and the Giant Stream. Long-slit spectra were obtained for a carefully selected sample of seven PNe using the OSIRIS spectrograph attached to the $10.4 \mathrm{~m}$ GTC in La Palma. Careful data reduction was carried out to produce the highest quality 1D spectra ever taken for M31 PNe in the optical range $(\sim 3600-7800 \AA)$. The temperature-sensitive [O III] $\lambda 4363$ auroral line was well detected in the spectra of all targets and, in most cases, the $[\mathrm{N} \mathrm{II}] \lambda 5755$ auroral line was also detected, enabling determination of the electron temperature. Electron densities were derived from the [Ar IV] and [S II] nebular doublets. We also estimated temperatures from the He I optical recombination line ratios, the first attempt of such effort for extragalactic PNe. The He I temperatures are generally lower than those derived from the forbidden lines, consistent with observations in the Galactic PNe.

Ionic abundances were derived based on the [O III] electron temperatures. Elemental abundances of oxygen, nitrogen, neon, sulfur and argon were estimated using the ICF method. The N/O ratios of our sample, including the three Northern Spur PNe in Paper I, are in the range $0.10-0.43$, and the $\mathrm{He} / \mathrm{H}$ ratios are $\sim 0.088-0.126$, indicating that they are Type II PNe, i.e., their progenitors probably have relatively low masses $\left(\leq 2 M_{\odot}\right)$. Using the empirical linear fit of the extinction versus core mass relation Kwitter et al. (2012), we roughly estimated that the core masses of our PN sample are in the range 0.59-0.65 $M_{\odot}$. Using the stellar evolution model, we estimated the progenitors' main-sequence lifetimes of our PNe might be as old as $\lesssim 2$ Gyr. We reckon that our core masses as well as the main-sequence lifetimes are highly uncertain. With the availability of deep GTC spectra to be obtained for more PNe in the substructures of M31, we will carry out detailed photoionization modeling for our sample, and much more robust core masses will be derived. Our sample has an almost homogeneous distribution of the oxygen abundances.

Our GTC sample includes a target that is so far the most distant $(\sim 3.65) \mathrm{PN}$ from the galaxy center ever discovered in M31. It was newly captured and identified by the LAMOST spectroscopic survey, and is the first PN discovered in the outer streams of M31. This $\mathrm{PN}$ is both spatially and kinematically related to the $\mathrm{Gi}$ ant Stream. The other six PNe targeted by GTC are three PNe in the Northern Spur and three associated with the Giant Stream. Our GTC run marks the first effort to carry out spectroscopic observations of PNe associated with the Giant Stream substructure. All the ten substructure-associated targets (including the three Northern Spur PNe in Paper I) are also well associated with the stellar orbit/streams that was proposed by Merrett et al. (2003) to connect the Northern Spur and the Giant Stream, both spatially and kinematically.

The abundance-ratio distribution of our sample suggests that they belong to the same old population, while their spatial and kinematical distribution hints at the possibility that Northern Spur and the Giant Stream have the same origin, i.e., both of the two substructures are the debris of the tidal interaction between M31 and its satellite(s). This postulation agrees with an estimate of the old age for our targets, given that galactic interactions have long timescales. Judging from the spatial 
positions and kinematics of PNe therein, we emphasize the hypothesis of Merrett et al. (2003) that the dwarf galaxy M32 (and/or others) might be responsible for the stellar streams and thus the origin of the two substructures. Deep spectroscopy of PNe in M32 will help to assess this postulation.

This work, together with Paper I, is a pioneering effort to study the substructures in M31 using PNe as tracers of chemistry. Detailed spectroscopy using high-quality spectra obtained at a $10 \mathrm{~m}$-class telescope has proved to be very successful in deriving accurate abundance ratios, constraining stellar populations and thus the possible origin of substructures. Effort as such may be extended to other Local Group galaxies.

\section{ACKNOWLEDGEMENTS}

This work is based on observations made with the Gran Telescopio Canarias (GTC), installed at the Spanish Observatorio del Roque de los Muchachos of the Instituto de Astrofísica de Canarias, in the island of La Palma. X.F. and M.A.G. acknowledge support from Spanish MICINN (Ministerio de Ciencia e Innovación) grant AYA 2014-57280-P co-funded with
FEDER funds. R.G.B. acknowledges support from the MICINN AYA2010-15081 grant. Y.Z. thanks the Hong Kong General Research Fund (HKU7062/13P) for the financial support of this study. The support and advice from the GTC staff, especially the GTC OSIRIS instrumental specialist, Antonio Cabrera-Lavers, is gratefully acknowledged. We thank Enrique Pérez Jiménez from the Instituto de Astrofísica de Andalucía (IAA-CSIC) for comments and suggestions. We also thank Michael R. Merrifield from the University of Nottingham for giving us the permission to reproduce the stellar orbit in Figure 7/ of this paper, based on Figure 2 of Merrett et al. (2003). We would also like to thank the anonymous referee whose comments have greatly improved the quality of this article. This research has made use of NASA's Astrophysics Data System (http://adsabs.harvard.edu), the SDSS DR10 Science Archive Server (SAS; http://data.sdss3.org/) and the SIMBAD Astronomical Database (http://simbad.ustrasbg.fr/simbad/).

Facilities: GTC (OSIRIS)

\section{REFERENCES}

Asplund, M., Grevesse, N., Sauval, A.-J., \& Scott, P. 2009, ARA\&A, 47, 481

Balick, B., Kwitter, K. B., Corradi, R. L. M., \& Henry, R. B. C. 2013, ApJ, 774, 3

Bell, K. L., Hibbert, A., \& Stafford, R. P. 1995, Phys. Scr., 52, 240

Benjamin, R. A., Skillman, E. D., \& Smits, D. P. 1999, ApJ, 514, 307

Benjamin, R. A., Skillman, E. D., \& Smits, D. P. 2002, ApJ, 569, 288

Bernard, E. J., Ferguson, A. M. N., Richardson, J. C., et al. 2015, MNRAS, 446, 2789

Biémont, E., \& Hansen, J. E. 1986, Phys. Scr., 34, 116

Bowen, I. S. 1935, ApJ, 81, 1

Caldwell, N., Morrison, H., Kenyon, S. J., et al. 2010, AJ, 139, 372

Cardelli, J. A., Clayton, G. C., \& Mathis, J. S. 1989, ApJ, 345, 245

Catalán, S., Isern, J., Carcía-Berro, E., \& Ribas, I. 2008, MNRAS, 387,1693

Ciardullo, R., \& Jacoby, G. H. 1999, ApJ, 515, 191

Clayton, G. C., Gordon, K. D., Bianchi, L. C., et al. 2015, ApJ, in press (arXiv: 1510.06983)

Corbelli, E., Lorenzoni, S., Walterbos, R., Braun, R., \& Thilker, D. 2010, A\&A, 511, A89

Corradi, R. L. M., Kwitter, K. B., Balick, B., Henry, R. B. C., \& Hensley, K. 2015, ApJ, 807, 181

Davey, A. R., Storey, P. J., \& Kisielius, R. 2000, A\&AS, 142, 85

de Vaucouleurs, G. 1958, ApJ, 128, 465

Dufour, R. J., Kwitter, K. B., Shaw, R. A., et al. 2015, ApJ, 803, 23

Esteban, C., Peimbert, M., García-Rojas, J., et al. 2004, MNRAS, 355,229

Esteban, C., Bresolin, F., Peimbert, M., et al. 2009, ApJ700, 654

Fang, X., \& Liu, X.-W. 2013, MNRAS, 429, 2791

Fang, X., Zhang, Y., García-Benito, R., Liu, X.-W., \& Yuan, H.-B. 2013, ApJ, 774, 138 (Paper I)

Ferguson, A. M. N., Irwin, M. J., Ibata, I. A., Lewis, G. F., \& Tanvir, N. R. 2002, AJ, 124, 1452

Galavis, M. E., Mendoza, C., \& Zeippen, C. J. 1995, A\&AS, 111, 347

Grandi, S. A. 1976, ApJ, 206, 658

Henry, R. B. C., Kwitter, K. B., \& Balick, B. 2004, AJ, 127, 2284

Henry, R. B. C., Speck, A., Karakas, A. I., et al. 2012, ApJ, 749, 61

Herwig, F. 2005, ARA\&A, 43, 435

Hirata, R., \& Horaguchi, T. 1995, Atomic Spectral Line List, VizieR On-line Data Catalogue

Howarth, I. D., \& Adams, S. 1981, Program EQUIB, Univ. Coll. London, London

Huchra, J. P., Brodie, J. P., \& Kent, S. M. 1991, ApJ, 370, 495

Hurley-Keller, D., Morrison, H. L., \& Harding, P., 2004, ApJ, 616, 804

Ibata, R. A., Chapman, S., Ferguson, A. M. N., Lewis, G. F., Irwin, M., \& Tanvir, N. 2005, ApJ, 634, 287
Ibata, R. A., Irwin, M. J., Lewis, G. F., \& Stotle, A. 2001a, ApJ, $547, \mathrm{~L} 133$

Ibata, R. A., Irwin, M. J., Lewis, G. F., Ferguson, A. M. N., \& Tanvir, N. R. 2001b, Natur, 412, 49

Ibata, R. A, Lewis, G. F., McConnachie, A. W., et al. 2014, ApJ, 780,128

Ibata, R. A., Martin, N. F., Irwin, M. J., et al. 2007, ApJ, 671, 1591

Irwin, M. J., Ferguson, A. M. N., Ibata, R. A., Lewis, G. F., \& Tanvir, N. R. 2005, ApJ, 628, L105

Izotov, Y. I., \& Thuan, T. X. 1999, ApJ, 511, 639

Izotov, Y. I., Thuan, T. X., \& Guseva, N.' G. 2012, A\&A, 546, A122

Jacoby, G. H., \& Ciardullo, R. 1999, ApJ, 515, 169

Kaler, J. B. 1986, ApJ, 308, 322

Kaler, J. B., \& Lutz, J. H. 1985, PASP, 97, 700

Keenan, F. P., Hibbert, A., Ojha, P. C., \& Conlon, E. S. 1993 Phys. Scr., 48, 129

Kennicutt, R. C., Bresolin, F., \& Garnett, D. R. 2003, ApJ, 591, 801

Kingsburgh, R. L., \& Barlow, M. J. 1994, MNRAS, 271, 257

Kniazev, A. Y., Grebel, E. K., Zucker, D. B., \& Rix, H.-W. 2014 AJ, 147,16

Kwitter, K. B., \& Henry, R. B. C. 2001, ApJ, 562, 804

Kwitter, K. B., Henry, R. B. C., \& Milingo, J. B. 2003, PASP, 115, 80

Kwitter, K. B., Lehman, E. M. M., Balick, B., \& Henry, R. .B. C. 2012, ApJ, 753, 12

Kwitter, K. B., Méndez, R. H., Peña, M., et al. 2014, RMxAA, 50, 203

Landi, E., \& Bhatia, A. K. 2005, At. Data Nucl. Data Tables, 89, 195

Leisy, P., \& Dennefeld, M. 2006, A\&A, 456, 451

Lennon, D. J., \& Burke, V. M. 1994, A\&AS, 103, 273

Lewis, G. F., Braun, R., McConnachie, A. W., et al. 2013, ApJ, 763,4

Liu, X.-W. 2006, in Barlow, M. J., Méndez, R. H., eds, IAU Symp. 234, Planetary Nebulae in our Galaxy and Beyond. Cambridge Univ. Press, Cambridge, p. 219

Liu, X.-W. 2012, in York, D. G., Gingerich, O., Zhang S.-N., Harper C. L., eds, New Vision 400: Engaging Big Questions in Astronomy and Cosmology Four Hundred Years after the Invention of the Telescope, CRC Press, p. 103

Liu, X.-W., Barlow, M. J., Zhang, Y., Bastin, R. J., \& Storey, P. J. 2006, MNRAS, 368, 1959

Liu, X.-W., \& Danziger, I. J. 1993, MNRAS, 263, 256

Liu, X.-W., Storey, P. J., Barlow, M. J., \& Clegg, R. E. S. 1995, MNRAS, 272, 369

Liu, X.-W., Storey, P. J., Barlow, M. J., Danziger, I. J., Cohen, M. \& Bryce, M. 2000, MNRAS, 312, 585

Majewski, S. R., Skrutskie, M. F., Weinberg, M. D., \& Ostheimer, J. C. 2003, ApJ, 599, 1082

Mateo, M. 1998, ARA\&A, 36, 435 
McConnachie, A. W., Irwin, M. J., Ferguson, A. M. N., et al. 2005, MNRAS, 356, 979

McConnachie, A. W., Irwin, M. J., Ibata, R. A., et al. 2003, MNRAS, 343, 1335

McConnachie, A. W., Irwin, M. J., Ibata, R. A., et al. 2009, Natur, 461,66

McConnachie, A. W., Irwin, M. J., Lewis, G. F., et al. 2004, MNRAS, 351, L94

McLaughlin, B. M., \& Bell, K. L. 2000, J. Phys. B: At. Mol. Opt. Phys., 33, 597

McNabb, I. A., Fang, X., Liu, X.-W., Bastin, R. J., \& Storey, P. J. 2013, MNRAS, 428, 3443

Mendoza, C. 1983, in IAU Symp. 103, Planetary Nebulae, ed. Flower, D., Reidel, Dordrecht, p.143

Mendoza, C., \& Zeippen, C. J. 1982a, MNRAS, 199, 1025

Mendoza, C., \& Zeippen, C. J. 1982b, MNRAS, 198, 127

Merrett, H. R., Kuijken, K., Merrifield, M. R., et al. 2003, MNRAS, 346, L62

Merrett, H. R., Merrifield, M. R., Douglas, N. G., et al. 2006, MNRAS, 369, 120

Milingo, J. B., Henry, R. B. C., \& Kwitter, K. B. 2002, ApJS, 138 285

Milingo, J. B., Kwitter, K. B., Henry, R. B. C., \& Souza, S. P. 2010, ApJ, 711, 619

Morrison, H. L., Harding, P., Hurley-Keller, D., \& Jacoby, G. 2003, ApJ, 597, L183

Newton, K., \& Emerson, D. T. 1977, MNRAS, 181, 573

Oke, J. B. 1974, ApJS, 27, 21

Oke, J. B. 1990, AJ, 99, 162

Osterbrock, D. E., \& Ferland, G. J. 2006, Astrophysics of Gaseous Nebulae and Active Galactic Nuclei (Sausalito, CA: Univ. Science Books)

Peimbert, M. 1971, Bol. Obs. Tonantzintla y Tacubaya, 6, 29

Porter, R. L., Ferland, G. J., Storey, P. J., \& Detisch, M. J. 2012, MNRAS, 425, L28

Pottasch, S. R., \& Bernard-Salas, J. 2006, A\&A, 457, 189
Pradhan, A. K., Montenegro, M., Nahar, S. N., \& Eissner, W. 2006 , MNRAS, 366, L6

Ramsbottom, C. A., Bell, K. L., \& Keenan, F. P. 1997, MNRAS, 284, 754

Ramsbottom, C. A., Bell, K. L., \& Stafford, R. P. 1996, At. Data Nucl. Data Tables, 63, 57

Richer, M. G., Stasińska, G., \& McCall, M. L. 1999, A\&AS, 135, 203

Robbins, R. R. 1968, ApJ, 151, 511

Sanders, N. E., Caldwell, N., McDowell, J., \& Harding, P. 2012 , ApJ, 758, 133

Savage, B. D. \& Mathis, J. S. 1979 ARA\&A, 17, 73

Schaller, G., Schaerer, D., Meynet, G., \& Maeder, A. 1992, A\&AS, 96, 269

Schlegel, D. J., Finkbeiner, D. P.. \& Davis, M. 1998, ApJ, 500, 525

Shaw, R. A., Lee, T.-H., Stanghellini, L. et al. 2010, ApJ, 717, 562

Stafford, R. P., Bell, K. L., Hibbert, A., \& Wijesundera, W. P. 1994, MNRAS, 268, 816

Stanghellini, L., Magrini, L., Villaver, E., \& Galli, D. 2010, A\&A, 521, A3

Storey, P. J., \& Hummer, D. G. 1995, MNRAS, 272, 41

Storey, P. J., \& Zeippen, C. J. 2000, MNRAS, 312, 813

Su, D.-Q., Cui, X.-Q., Wang, Y., \& Yao, Z. 1998, Proc. SPIE, 3352

Tayal, S. S., \& Gupta, G. P. 1999, Ap.J, 526, 544

Vassiliadis, E., \& Wood, P. R. 1994, ApJS92, 125

White, S. D. M. 1978, MNRAS, 184, 185

White, S. D. M., \& Rees, M. J. 1978, MNRAS, 183, 341

Wofford, A. 2009, MNRAS, 395, 1043

Yuan, H.-B., Liu, X.-W., Huo, Z.-Y., et al. 2010, RAA, 10, 599

Zeippen C. J. 1987, A\&A, 173, 410

Zhang, Y., Liu, X.-W., Liu, Y., \& Rubin, R. H. 2005, MNRAS 358,457

Zurita, A., \& Bresolin, F. 2012, MNRAS, 427, 1463 